\title{
Towards a Definition of Locality in a Manifoldlike Causal Set
}

\author{
Lisa Glaser $^{a}$ and Sumati Surya ${ }^{b}$ \\ ${ }^{a}$ Niels Bohr Institute, Copenhagen, Denmark \\ ${ }^{b}$ Raman Research Institute, Bangalore, India
}

15th October 2018

\begin{abstract}
It is a common misconception that spacetime discreteness necessarily implies a violation of local Lorentz invariance. In fact, in the causal set approach to quantum gravity, Lorentz invariance follows from the specific implementation of the discreteness hypothesis. However, this comes at the cost of locality. In particular, it is difficult to define a "local" region in a manifoldlike causal set, i.e., one that corresponds to an approximately flat spacetime region. Following up on suggestions from previous work, we bridge this lacuna by proposing a definition of locality based on the abundance of $m$-element order-intervals as a function of $m$ in a causal set. We obtain analytic expressions for the expectation value of this function for an ensemble of causal set that faithfully embeds into an Alexandrov interval in $d$-dimensional Minkowski spacetime and use it to define local regions in a manifoldlike causal set. We use this to argue that evidence of local regions is a necessary condition for manifoldlikeness in a causal set. This in addition provides a new continuum dimension estimator. We perform extensive simulations which support our claims.
\end{abstract}

\section{Introduction}

Causal set theory is a candidate for quantum gravity where the spacetime continuum is replaced by a discrete substructure which is a locally finite partially ordered set [1, 2, 3, 4]. It is often assumed that Lorentz violation is an inevitable consequence of spacetime discreteness. This is explicitly false in a causal set discretisation of a spacetime - on the contrary, as shown in [5], the causal set hypothesis instead implies Lorentz invariance. However, this comes at the cost of locality. In a causal set, the nearest neighbours of an element are the links or irreducible relations. For example, in infinite causal set that is approximated by Minkowski spacetime, every element has an infinite number of nearest neighbours, both to the past and to the future. The resulting graph is therefore of infinite valency, in stark contrast to other types of spacetime discreteness in which the graphs are of finite valency. Indeed, it is this very feature of a causal set which captures the essence of Lorentz-invariant discreteness, since there are non-compact invariant hyperbolae associated with every Lorentz boost about a spacetime event in Minkowski spacetime. This feature of causal set discretisation in turn is due to the requirement of a uniform distribution which preserves the number to volume correspondence, crucial to the recovery of the Lorentzian spacetime geometry in the continuum approximation [5]. 
While Lorentz invariance is a great asset to causal set theory, the resulting non-locality of the causal set graph impedes a straightforward reconstruction of continuum information from the discrete substructure. Unlike a simplicial decomposition, for example, where the discrete scalar curvature has a simple local geometric interpretation, there is no analogous local construction in a causal set. Indeed, it is only recently that a causal set expression for scalar curvature and hence a causal set action has been found in arbitrary dimensions [6, 7]. Nevertheless, despite the difficulty in recovering local information from a causal set, over the years substantial progress has been made in understanding how topology and geometry emerges from a causal set, sometimes with the aid of fairly ingenious order-theoretic constructions. This includes the reconstruction of spacetime dimension, time-like distance, space-like distance and spatial homology, for causal sets that are approximated by continuum spacetimes [8].

Importantly, in many of these reconstructions the causal set is assumed to be approximated by a region of curved spacetime which is small compared to the scale of flatness. In the continuum such a region has a natural interpretation of being "local" or approximately flat. From the continuum perspective small, or local neighbourhoods are essential to several geometric constructions and are key to the conception of a manifold. However, until now there has been no purely order theoretic characterisation of such local neighbourhoods in a causal set. It is therefore an important step to be able to define local regions in a causal set and hence provide an appropriate context for some of the reconstruction results.

Our prescription for locality uses a well known order theoretic definition of a spacetime region, namely an Alexandrov interval $I[x, y]:=\{z \mid x \prec \prec z \prec \prec y\}$, where $\prec \prec$ is the chronological relation. In the continuum $I[x, y]$ is characterised both by the time-like distance $\tau(x, y)$ from $x$ to $y$, as well as its volume $\operatorname{Vol}(I[x, y])$. Unlike open ball neighbourhoods in a Riemannian manifold, however, even arbitrarily small choices of $\tau(x, y)$ or $\operatorname{Vol}(I[x, y])$ do not correspond to a region in which the scale of flatness is large as illustrated in Fig. 1. In the continuum, $\tau(x, y) \operatorname{or} \operatorname{Vol}(I[x, y])$ are the only Lorentz invariant quantities that characterise $I[x, y]$. However, it is clear that the corresponding discrete geometry, i.e., a causal set which faithfully embeds into $I[x, y]$, should contain more detailed geometric information.

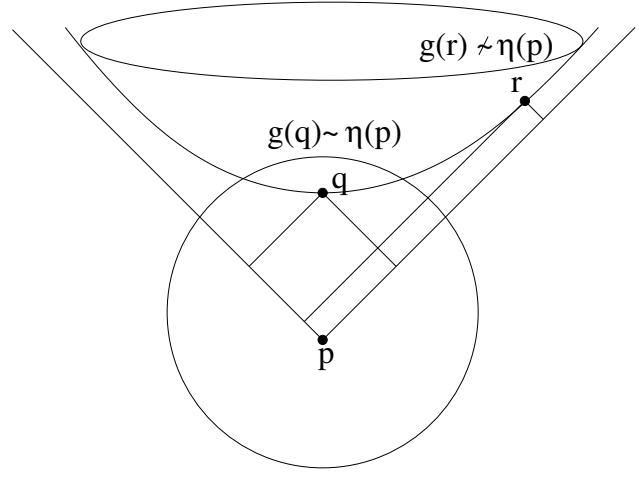

Figure 1: The two Alexandrov intervals $I[p, q]$ and $I[p, r]$ have the same volume, but the former lies in an approximately flat neighbourhood of $p$, while the latter does not. 
The main proposal of our work is that there is indeed an order theoretic characterisation of locality. The motivation for this arises from the work of Benincasa and Dowker [6, 7] where it was found that the scalar curvature of an element $e$ in a causal set $C$ can be obtained from knowing the abundances $N_{m}$ of order-intervals of size $m$ that lie to the past of $e$. Here, $N_{0}$ is the abundance of 0 order-intervals, i.e., the number of irreducible relations or links in $C, N_{1}$ the abundance of 1-element order intervals, or irreducible 3-chains etc.For a generic non-locality scale the discrete Einstein-Hilbert action is constructed from all possible $N_{m}$, but when the non-locality scale is taken to be the Planck scale, the action simplifies considerably. For example, the $2 \mathrm{~d}$ causal set action takes the elegant form

$$
\frac{1}{\hbar} S_{2 D}=N-2 N_{0}+4 N_{1}-2 N_{2}
$$

which only involves the abundances of intervals of volume 0,1 and 2 .

In simulations of $2 \mathrm{~d}$ quantum gravity using Markov Chain Monte Carlo methods the $N_{m}$ were used as covariant observables for tracking thermalisation [9]. Importantly, it was observed that $N_{m}$ as a function of $m$ has a characteristic behaviour in the phase in which flat spacetime is emergent, and differs drastically from the non-manifold phase. Simulations of $2 d$ flat spacetime showed that this characteristic curve could indeed be used as a reliable indicator of flatness.

In this work we carry this idea forward. We begin by obtaining analytic expressions for the expectation value of the interval abundances $\left\langle N_{m}^{d}\right\rangle$ for a causal set that faithfully embeds into an Alexandrov interval in flat spacetime of arbitrary dimensions $d$. Our main proposal is that the characteristic curves for $\left\langle N_{m}^{d}\right\rangle$ as a function of $m$ can be used to define a local region in a manifoldlike causal set $C$. The existence of a local region in a causal set is therefore a necessary condition for manifoldlikeness of $C$ and hence a new continuum dimension estimator. Specifically, since the characteristic curves for $\left\langle N_{m}^{d}\right\rangle$ for fixed cardinality are sufficiently distinct for each $d$, it is possible to use them to find the continuum dimension of the local region in the causal set . This estimator therefore gives a null result for causal sets which are non-manifoldlike. Because the $\left\langle N_{m}^{d}\right\rangle$ provide an entire family of observables, it is tempting to conjecture that the requirement on interval abundances is not only a necessary but also a sufficient requirement for manifoldlikeness of a causal set.

We test our proposals with simulations of causal sets that are approximated by spacetimes as well as those that are not. We find that our necessary condition for manifoldlikeness works extremely well even for relatively small causal sets.

Indeed, not only do the simulated interval abundances reproduce on average the characteristic curve, they follow it with reasonable precision even in a single realisation.

The latter is especially important in assessing manifoldlikeness in a single causal set, as opposed to an ensemble of causal sets. Our simulations verify that apart from being able to determine the local regions of a manifold like causal set, our prescription is also a test for manifoldlikeness itself and thence, manifold dimension.

Our construction demonstrates clearly the geometric richness of a locally finite poset which is approximated by a spacetime. Our analysis indicates the existence of a local geometric "rigidity" present in manifold like causal sets - significant deviations from the $\left\langle N_{m}^{d}\right\rangle$ result in Alexandrov intervals that are explicitly not local. Thus, $\left\langle N_{m}^{d}\right\rangle$ as function of $m$ provides us a local, covariant, geometric measure for manifoldlikeness.

In Section 2 we give a short introduction to the main concepts of causal set theory and define quantities that we will need further on. The calculation of the interval abundances in flat spacetime are in Section 3. We find that the ratio of these abundances is scale invariant in the large $N$ 
limit, which provides a strong motivation for using the $\left\langle N_{m}^{d}\right\rangle$ as indicators of locality. In Section 4 we present the main ideas in this work, namely how the $\left\langle N_{m}^{d}\right\rangle$ can be used to define locality in a manifoldlike causal set. We then conjecture that the $\left\langle N_{m}^{d}\right\rangle$ provide a rigidity criterion for manifoldlikeness for a causal set that faithfully embeds into an Alexandrov interval in flat spacetime. In Section 5 we present results from extensive numerical simulations that support these ideas. In particular, we examine the interval abundances of causal sets that we know a priori to be either manifoldlike or not and find that our test works extremely well. Our tests include flat spacetimes of different dimensions, the 2-d cut-trousers topology, as well as the flat geometries on $S^{1} \times \mathbb{R}$ and $T^{2} \times \mathbb{R}, 4$-d FRW spacetimes, including deSitter spacetime, and some examples of non-manifoldlike causal sets. In particular, we examine the claim from [10] that causal sets grown with transitive percolation are manifoldlike. We find that while macroscopic indicators may suggest manifoldlikeness, it fails our microscopic test. We end with a discussion on open questions and future directions in Section 6 .

\section{Preliminaries}

Studies of Lorentzian geometry have long stressed the importance of the causal structure [1]. For causal spacetimes, the causal structure provides a partial order on the set of spacetime events. This partial order is a unique characteristic of a Lorentzian signature $((-,+,+\ldots+))$ spacetime, a feature absent in all other signature spacetimes. It was shown by Malament, Hawking and others in 12 that a bijection between two past and future distinguishing spacetimes which preserves the causal structure is a conformal isomorphism. Thus, knowing the causal relations between all points in a spacetime is enough to define its geometry up to a conformal factor. The causal set hypothesis of a fundamental discreteness adds to this classical result by providing a discrete volume element to help recover the conformal factor. Roughly, every discrete event comes with approximately one unit of spacetime volume so that the number of events in a region corresponds to the volume of that region. In other words, in causal set theory, an appropriately discretised partially ordered set replaces continuum Lorentzian geometry, summarised in the slogan: Order + Number $\approx$ Spacetime.

Formally a causal set $C$ is defined to be a locally finite partially ordered set, namely a countable set $C$ with an order relation $\preceq$ on its elements which is

(a) Reflexive: for all $x \in \mathcal{C}, x \preceq x$

(b) Transitive: for all $x, y, z \in \mathcal{C}$ and $x \preceq y$ and $y \preceq z$ then $x \preceq z$,

(c) Acyclic: for all $x, y \in \mathcal{C}, x \preceq y \preceq x \Rightarrow x=y$

(d) Locally Finite: for all $x, y \in \mathcal{C}|I(x, y)| \equiv|\{z \mid x \preceq z \preceq y\}|<\infty$.

This last condition is equivalent to the assumption of a fundamental discreteness. The first figure in Fig. 2 shows the Hesse diagram of a small causal set where the elements are numbered and the links are denoted by arrows.

Causal set quantum gravity is thus a quantum theory of causal sets with the continuum existing only as an approximation to a fundamentally discrete substructure. In particular, the ensemble of causal sets that are approximated by a given spacetime $(M, g)$ is obtained via a Poisson process 


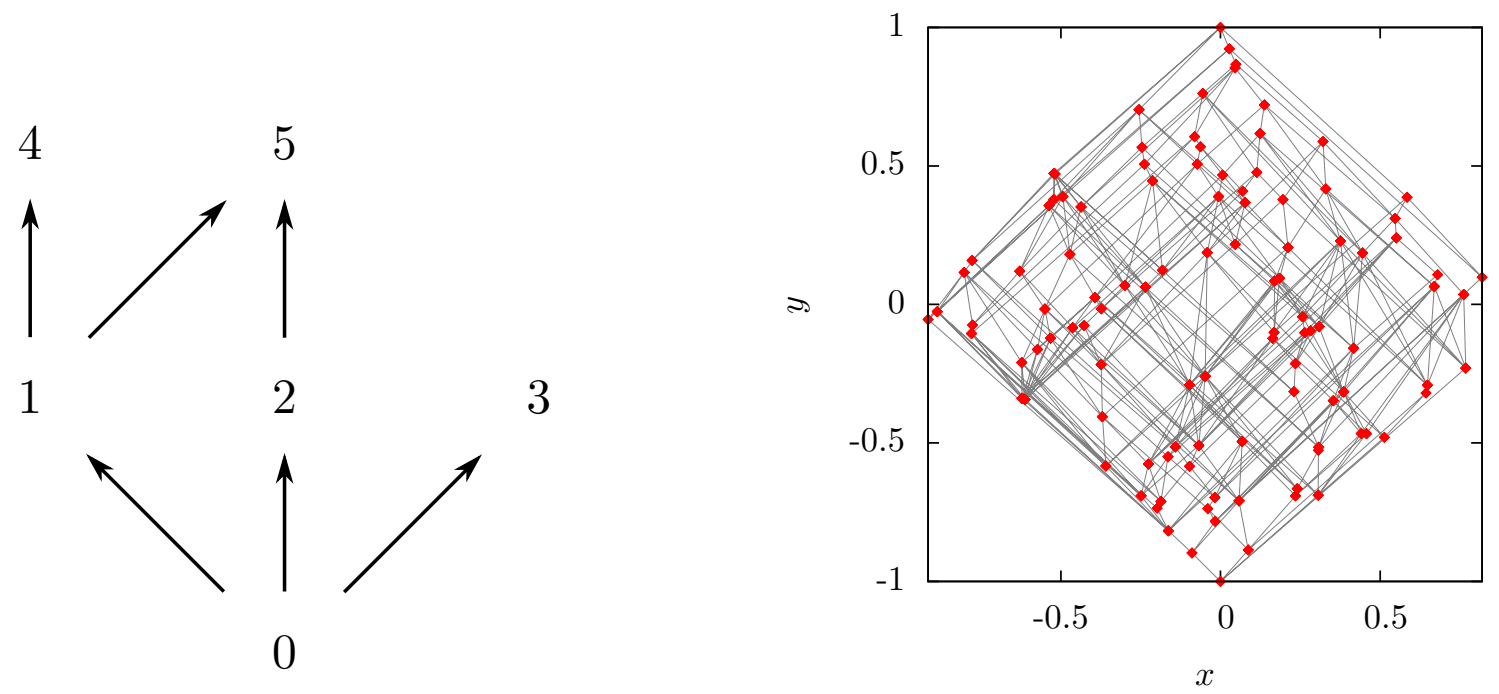

Figure 2: On the left is the Hesse diagram of a simple causal set where only the links are shown by arrows. The figure on the right is a sprinkling of 100 elements into an interval of flat spacetime, where all the causal relations are depicted by lines.

for a given discreteness scale $\rho^{-1}$. The probability of assigning $m$-elements of a causal set $C$ in a spacetime region of volume $V$ is given by

$$
P_{V}(m)=\frac{(\rho V)^{m}}{m !} e^{-\rho V}
$$

The causal set is then recovered via the induced causal relations on the set of elements. This Poisson "sprinkling" is a key feature of causal set discretisation of the continuum. The second figure in Fig. 2 shows a sprinkling of 100 elements into flat space. Conversely, given a causal set, $C$, we say that it is approximated by a spacetime $(M, g)$ if there exists a faithful embedding $\Phi: C \rightarrow(M, g)$ such that $\Phi(C) \subset(M, g)$ corresponds to a high probability Poisson sprinkling into $(M, g)$ with the order relations in $C$ being the same as those induced by the causal relations in $(M, g)$ onto $\Phi(C)$. An important conjecture in the theory is that a given causal set cannot faithfully embed into two distinct spacetimes, namely, those which differ on scales larger than the discreteness scale. In other words continuum structures below the discreteness scale are irrelevant to the theory. We refer the reader to the literature for more details on the basics of the causal set hypothesis [1, 2, 3, 4,

As discussed in the introduction, the key focus of this work is to be able to define a local region in a manifoldlike causal set. In the continuum, an Alexandrov interval $I[x, y] \equiv\{z \mid x \prec \prec z \prec \prec y\}$, where $\prec \prec$ is the chronological relation. In a causal set, there is no a priori distinction between causal and chronological relations and hence we define an order interval $I[x, y] \subset C$ as $I[x, y]=$ $\{z \mid x \prec z \prec y\}$. A natural characterisation of $I[x, y]$ is its cardinality or discrete volume. However, this information does not suffice to distinguish an $I[x, y]$ which is local and one that is not. Since the discrete geometry should include all relevant information about the continuum, we expect that there must exist other observables in $I[x, y]$ which can be used to characterise locality. In this work, we find that the abundance of $m$-element order-intervals in $I[x, y]$ is indeed such a family of 


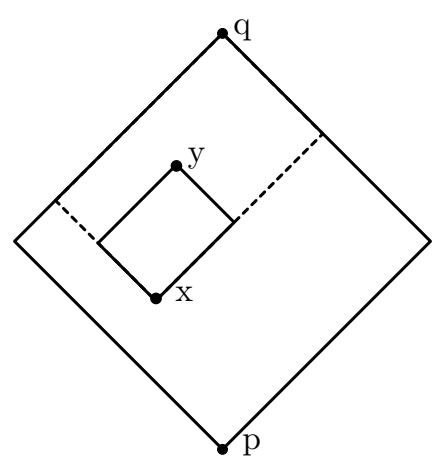

Figure 3: The Alexandrov neighbourhood $I[p, q]$ in the integration Eqn.(4): $x$ lies anywhere in $I[p, q]$ while $y$ lies in the intersection of $I[p, q]$ with the chronological future of $x$.

observables. Namely for every $m$ we count the number or abundance of order intervals of size $m$ in $I[x, y]$. For us, an $m=0$ order interval is a link, namely an order interval which contains only its end points, an $m=1$ order interval is one with a single element that lies between the end points, or an irreducible 3 chain, and $m=2$ can be an irreducible 4 chain or an irreducible diamond poset, i.e., an order interval with two elements between the end points.

For a causal set that faithfully embeds into flat spacetime, we find that the interval abundances follow a characteristic, monotonically decreasing curve as $m$ increases. It is this characteristic curve that we will use as a "ruler" to determine the locality of an order interval in a more general manifoldlike causal set. However, in order to do so, we would like to ensure that the scale of flatness in every region of the spacetime is much larger than the discreteness scale, so that the manifold approximation of the causal set is well-defined everywhere. By this we mean the following. Consider a causal set that faithfully embeds into an "approximately flat" spacetime region in which Riemann normal coordinates are valid. Such a region is characterised by a dimensionless size which we will refer to as the scale of flatness $\zeta>>^{-1}>>1$ where $\zeta=R \tau^{2}$ with $R$ denoting any component of the Riemann tensor at an event in the region and $\tau$ the proper time between any two events in the region. In flat spacetime $\zeta=0$ and hence the size $\zeta^{-1} \rightarrow \infty$ as expected. For a generic spacetime we will refer to such regions as "small": for a given $R$, the size of the region $\tau$ must be small enough for $\zeta<<1$. Let $C$ be a causal set that faithfully embeds at density $\rho$ into an Alexandrov interval $I[x, y]$ of volume $V$ which lies in a region for which $\zeta^{-1}>>1$. If $N \sim \rho V \sim 1$ then the continuum approximation of $C$ breaks down. Thus, in order for the $I[x, y]$ to be adequately represented by the causal set, we require that $N>>1$, i.e., the discreteness scale $N^{-1}$ must be small with respect to the scale of flatness $\zeta^{-1}$.

\section{The Abundances of Order Intervals in Flat Spacetime}

We now find closed form expressions for the abundance of order-intervals in a causal set $C$ that is faithfully embedded into an Alexandrov interval $I[p, q]$ in flat spacetime. To begin with we find the abundance of links $\left\langle N_{0}^{d}\right\rangle$. Lorentz invariance then allows us to generalise this expression to that for $\left\langle N_{m}^{d}\right\rangle$ in a straightforward way. Although we use series expansions to evaluate the integrals, the final expressions take relatively simple closed forms.

Consider the interval $I[p, q]$ in Fig. 3 with volume $V$ and proper time $\tau$ from $p$ to $q$. The 
probability that there is a link from an element $x$ to an element $y$ in this region is given by

$$
P_{x y}=e^{-\rho V_{x y}},
$$

where $V_{x y}$ is the spacetime volume of the Alexandrov interval $I[x, y] \subset I[p, q]$ in the embedding spacetime. Moreover, given $x, y$ lies to its future, i.e., $y \in I[x, q]$, while $x$ can lie anywhere in $I[p, q]$. Thus, the expectation value for the number of links in $I[p, q]$ is given by

$$
\left\langle N_{0}^{d}(\rho, V)\right\rangle=\rho^{2} \int_{\diamond} \mathrm{d} V_{x} \int_{\diamond_{x}} \mathrm{~d} V_{y} e^{-\rho V_{x y}},
$$

where the symbol $\diamond$ denotes $I[p, q]$ and the symbol $\diamond_{x}$ denotes $I[x, q]$. Since this expression is Lorentz invariant, it can only depend on the proper time $\tau$ or volume $V$ of $I[p, q]$. Thus we may choose $p$ at the origin $p=(0, \ldots, 0)$ and $q$ on the time axis $q=(\sqrt{a}, 0, \ldots, 0)$, where $\tau=\sqrt{2} a$.

Lorentz covariance also implies that the integration over $\diamond_{x}$ depends only the proper time $\tau(x, q)$ and the volume $V_{x q}$ of $I[x, q]$. We may thus again calculate this integral in convenient coordinates and then recast it in terms of $\tau(x, q)$ and $V_{x q}$. We take $x$ to lie at the origin $x=(0, \ldots, 0)$ and $q$ to lie on the time axis $q=\left(\sqrt{2} a^{\prime}, 0, \ldots, 0\right)$, where $\tau(x, q)=\sqrt{2} a^{\prime} \leq \sqrt{2} a$. Using lightcone coordinates

$$
v=\frac{1}{\sqrt{2}}(t+r) \quad u=\frac{1}{\sqrt{2}}(t-r),
$$

the integration measure in flat space for $y=\left(u_{y}, v_{y}, \vec{\Omega}_{y}\right)$ is then

$$
\int \mathrm{d} V_{y}=\frac{1}{2^{\frac{d}{2}-1}} \int \mathrm{d} \Omega_{y} \int_{0}^{a^{\prime}} \mathrm{d} v_{y} \int_{0}^{v} \mathrm{~d} u_{y}(v-u)^{d-2} .
$$

The function we will integrate over does not involve the angular coordinates of $y$, since $\tau(x, y)=$ $\sqrt{2 u_{y} v_{y}}$. We can then rewrite

$$
\rho V_{x y}=\rho \frac{S_{d-2} 2^{-\frac{d}{2}+1}}{d(d-1)}\left(u_{y} v_{y}\right)^{\frac{d}{2}}=\rho \zeta_{d}\left(u_{y} v_{y}\right)^{\frac{d}{2}},
$$

where $S_{d-2}$ is the volume of the $d-2$ sphere $S_{d-2}=\int \mathrm{d} \Omega_{y}=\frac{2 \frac{d-1}{2}}{\Gamma\left(\frac{d-1}{2}\right)}$, and we define the dimension dependent constant $\zeta_{d} \equiv \frac{S_{d-2} 2^{-\frac{d}{2}+1}}{d(d-1)}$. Thus the angular integration over $\diamond_{x}$ factors out so that, in these coordinates, the integral $I_{\diamond_{x}}^{\prime} \equiv \int_{\diamond_{x}} \mathrm{~d} V_{y} e^{-\rho V_{x y}}$ reduces to

$$
I_{\diamond_{x}}^{\prime}=d(d-1) \zeta_{d} \int_{0}^{a^{\prime}} \mathrm{d} v \int_{0}^{v} \mathrm{~d} u(v-u)^{d-2} e^{-\rho \zeta_{d}(u v)^{d / 2}},
$$

where we have suppressed the subscripts in $\left(u_{y}, v_{y}\right)$. Expanding $(v-u)^{d-2}$ in terms of binomial coefficients and $e^{-\rho \zeta_{d}(u v)^{d / 2}}$ as a power series simplifies the integration considerably

$$
\begin{aligned}
I_{\diamond_{x}}^{\prime} & =d(d-1) \zeta_{d} \sum_{n=0}^{\infty} \frac{\left(-\rho \zeta_{d}\right)^{n}}{n !} \sum_{k=0}^{d-2}\left(\begin{array}{c}
d-2 \\
k
\end{array}\right)(-1)^{k} \int_{0}^{a^{\prime}} \mathrm{d} v \int_{0}^{v} \mathrm{~d} u v^{d\left(\frac{n}{2}+1\right)-2-k} u^{\frac{d n}{2}+k} \\
& =d(d-1) \zeta_{d} \sum_{n=0}^{\infty} \frac{\left(-\rho \zeta_{d}\right)^{n}}{n !} \frac{a^{\prime d(n+1)}}{d(n+1)} \sum_{k=0}^{d-2}\left(\begin{array}{c}
d-2 \\
k
\end{array}\right) \frac{(-1)^{k}}{\frac{d n}{2}+k+1} .
\end{aligned}
$$


Rewriting

$$
\sum_{k=0}^{d-2}\left(\begin{array}{c}
d-2 \\
k
\end{array}\right) \frac{(-1)^{k}}{\frac{d n}{2}+k+1}=\frac{\Gamma(d-1) \Gamma\left(\frac{d n}{2}+1\right)}{\Gamma\left(\frac{d}{2}(n+2)\right)}
$$

we find that

$$
I_{\diamond_{x}}^{\prime}=d(d-1) \zeta_{d} \Gamma(d-1) \sum_{n=0}^{\infty} \frac{\left(-\rho \zeta_{d}\right)^{n}}{n !} \frac{a^{\prime d(n+1)}}{d(n+1)} \frac{\Gamma\left(\frac{d n}{2}+1\right)}{\Gamma\left(\frac{d}{2}(n+2)\right)}
$$

We can now convert the above expression into a Lorentz covariant form, by substituting $a^{\prime}$ for the proper time $\tau(x, q)=\sqrt{2} a^{\prime}$ of $I[x, q]$. In the original coordinates adapted for $I[p, q]$ this is $\tau(x, q)^{2}=2\left(a-v_{x}\right)\left(a-u_{x}\right)$ Thus, to complete the calculation of $\left\langle N_{0}^{d}\right\rangle$ we must evaluate the integral

$$
I_{\diamond}=\int_{\diamond} \mathrm{d} V_{x}\left(\left(a-u_{x}\right)\left(a-v_{x}\right)\right)^{\frac{d(n+1)}{2}}=\zeta_{d} d(d-1) \int_{0}^{a} \mathrm{~d} v_{x} \int_{0}^{v_{x}} \mathrm{~d} u_{x}\left(v_{x}-u_{x}\right)^{d-2}\left(\left(a-u_{x}\right)\left(a-v_{x}\right)\right)^{\frac{d(n+1)}{2}} .
$$

To shorten the notation we defined this integral without the sum over $n$ which we will have to restore in the final expression for $\left\langle N_{0}^{d}\right\rangle$. Substituting $u=a-u_{x}$ and $v=a-v_{x}$ and again using the binomial expansion

$$
\begin{aligned}
I_{\diamond} & =\zeta_{d} d(d-1) \sum_{k=0}^{d-2}\left(\begin{array}{c}
d-2 \\
k
\end{array}\right)(-1)^{k} \int_{0}^{a} \mathrm{~d} v \int_{0}^{v} \mathrm{~d} u u^{\frac{d}{2}(n+1)+k} v^{\frac{d}{2}(n+3)-2-k} \\
& =\zeta_{d} d(d-1) \frac{a^{d(n+2)}}{d(n+2)} \sum_{k=0}^{d-2}\left(\begin{array}{c}
d-2 \\
k
\end{array}\right)(-1)^{k} \frac{1}{\frac{d}{2}(n+1)+k+1} .
\end{aligned}
$$

Using the identity (11), and the fact that $V=\zeta_{d} a^{d}$, we find the Lorentz covariant expression for the average number of links in an interval $I[p, q]$ of volume $V$ to be

$$
\left\langle N_{0}^{d}\right\rangle(\rho, V)=\Gamma(d)^{2} \sum_{n=0}^{\infty} \frac{(-\rho V)^{n+2}}{(n+2) !} \frac{\Gamma\left(\frac{d n}{2}+1\right)}{\Gamma\left(\frac{d}{2}(n+2)\right)} \frac{\Gamma\left(\frac{d}{2}(n+1)+1\right)}{\Gamma\left(\frac{d}{2}(n+3)\right)} .
$$

This expression can now be used to find the $m$ element interval abundances in flat space for general $m$ by observing that (2) can be rewritten as

$$
P(m, V, \rho)=\frac{(-\rho)^{m}}{m !} \frac{\partial^{m}}{\partial \rho^{m}} e^{-\rho V} .
$$

Thus, the average number of $m$-element intervals in a volume $V$ is simply given as

$$
\begin{aligned}
\left\langle N_{m}^{d}\right\rangle(\rho, V) & =\rho^{2} \int_{\diamond} \mathrm{d} v_{y} \int_{\diamond_{y}} \mathrm{~d} V_{x} \frac{(\rho V)^{m}}{m !} e^{-\rho V} \\
& =\frac{(-\rho)^{m+2}}{m !} \frac{\partial^{m}}{\partial \rho^{m}} \int_{\diamond} \mathrm{d} V_{x} \int_{\diamond_{y}} \mathrm{~d} v_{y} e^{-\rho V} \\
& =\frac{(-\rho)^{m+2}}{m !} \frac{\partial^{m}}{\partial \rho^{m}} \rho^{-2}\left\langle N_{0}^{d}\right\rangle(\rho, V)
\end{aligned}
$$




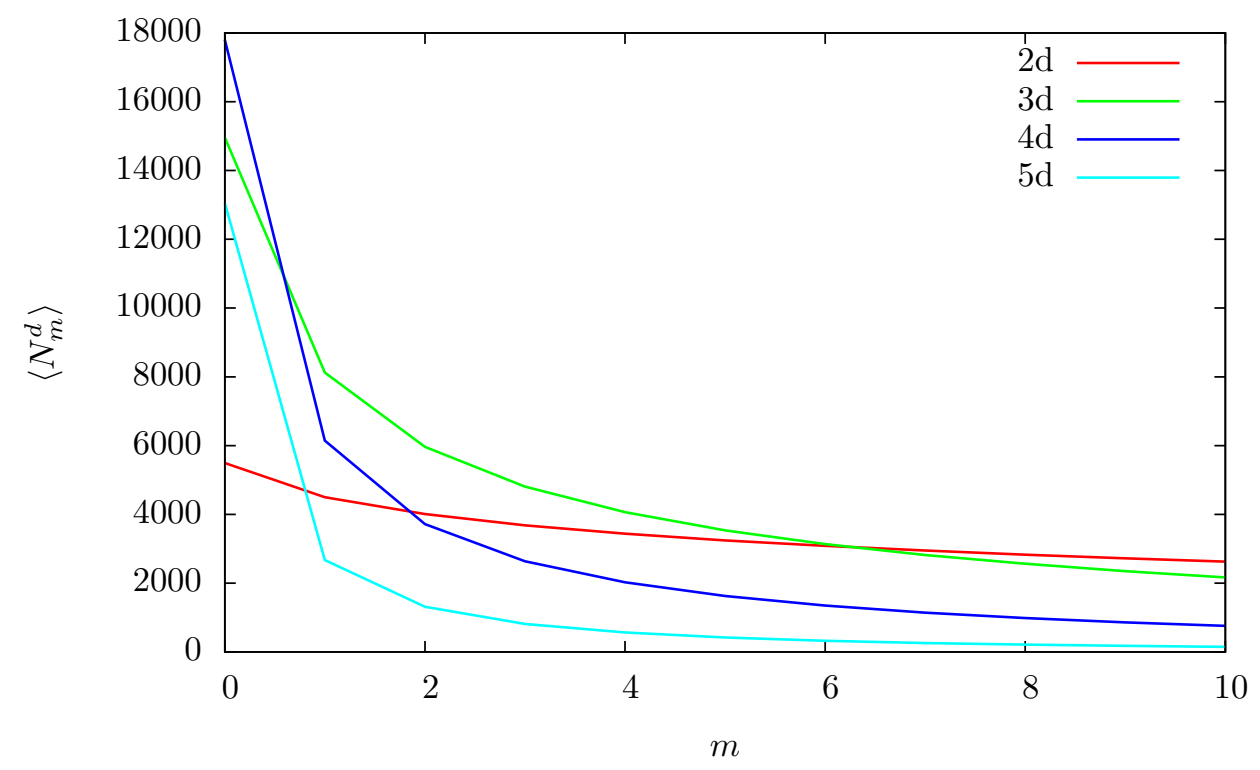

Figure 4: The function $\left\langle N_{m}^{d}\right\rangle \mathrm{v} / \mathrm{s} m$ for $N=1000$ and $d=1, \ldots, 5$.

Which evaluates to:

$$
\begin{aligned}
\left\langle N_{m}^{d}\right\rangle(\rho, V)= & \frac{\Gamma(d)^{2}}{m !}(-\rho V)^{m+2} \sum_{n=0}^{\infty} \frac{(-\rho V)^{n}}{n !} \frac{1}{(n+m+1)(n+m+2)} \times \\
& \frac{\Gamma\left(\frac{d}{2}(n+m)+1\right)}{\Gamma\left(\frac{d}{2}(n+m+2)\right)} \frac{\Gamma\left(\frac{d}{2}(n+m+1)+1\right)}{\Gamma\left(\frac{d}{2}(n+m+3)\right)} .
\end{aligned}
$$

This can then be recast as a closed form expression in terms of generalised hypergeometric functions:

$$
\begin{aligned}
\left\langle N_{m}^{d}\right\rangle(\rho, V)= & \frac{(\rho V)^{m+2}}{(m+2) !} \frac{\Gamma(d)^{2}}{\left(\frac{d}{2}(m+1)+1\right)_{d-1}} \frac{1}{\left(\frac{d}{2} m+1\right)_{d-1}} \\
& { }_{d} F_{d}\left(\begin{array}{c}
\left.1+m, \frac{2}{d}+m, \frac{4}{d}+m, \ldots, \frac{2(d-1)}{d}+m\right) \\
3+m, \frac{2}{d}+m+2, \frac{4}{d}+m+2, \ldots, \frac{2(d-1)}{d}+m+2
\end{array} \mid-\rho V\right),
\end{aligned}
$$

where ${ }_{p} F_{q}\left(\left\{a_{1}, \ldots, a_{p}\right\},\left\{b_{1}, \ldots, b_{q}\right\} \mid-z\right)$ is a generalised hypergeometric function and $(a)_{n}$ is the Pochhammer symbol. This expression is convergent because, as is well known, generalised hypergeometric functions converge for all $z$ values if $p \leq q$. The details of obtaining this form for the $\left\langle N_{m}^{d}\right\rangle$ are given in appendix A.

In Fig. 4 we plot the function $\left\langle N_{m}^{d}\right\rangle$ for different values of $d .\left\langle N_{m}^{d}\right\rangle$ rapidly and monotonically decreases as $m$ increases thus providing a clear characteristic signature for the flat spacetime case, which we will use to define locality and thence a continuum dimension estimator. 


\subsection{The Asymptotic Limit}

We now examine the behaviour of these expressions in the large $N=\rho V$ limit. Although the continuum limit is not physically relevant per se to causal set theory, it is nevertheless an interesting limit of the theory. Clearly, $\left\langle N_{m}^{d}\right\rangle$ will diverge with $N$, but it is not clear apriori what the behaviour will be after normalisation, say with respect to the abundance of links, $\left\langle N_{0}^{d}\right\rangle$. If there were a leading order $N$-dependence, then either this would diverge, or go to zero in the limit, thus drastically changing the nature of the $\left\langle N_{m}^{d}\right\rangle$ characteristic curve.

What we find is that the ratio is in fact independent of $N$ to leading order and yields a surprisingly simple expression in the asymptotic limit

$$
\mathcal{S}_{m}^{d} \equiv \lim _{\rho \rightarrow \infty} \frac{\left\langle N_{m}^{d}\right\rangle(\rho, V)}{\left\langle N_{0}^{d}\right\rangle(\rho, V)}=\frac{\Gamma\left(\frac{2}{d}+m\right)}{\Gamma\left(\frac{2}{d}\right) \Gamma(m+1)} .
$$

This scale invariance seems to echo that of Minkowski spacetime suggesting that the $\left\langle N_{m}^{d}\right\rangle$ captures an essential and perhaps even defining ingredient of flat spacetime geometry. We discuss this in some detail in the following section.

Here we give a quick sketch of how this limit is obtained, leaving details to appendix C. The $N=\rho V$ dependence in Eqn. 20 comes from the overall $N^{m+2}$ factor as well as the hypergeometric function ${ }_{d} F_{d}$ which when appropriately rearranged is of the form

$$
{ }_{d} F_{d}\left(\begin{array}{c}
a_{1}, \ldots, a_{d} \\
a_{1}+2, \ldots, a_{d}+2
\end{array} \mid-N\right), \quad a_{i}=\frac{2 i}{d}+m, i=1, \ldots d-1, \quad a_{d}=1+m .
$$

Thus, to investigate the $N \rightarrow \infty$ limit of the $\left\langle N_{m}^{d}\right\rangle$ we need a large $N$ expansion of this function. We make repeated use of the following identity [13]

$$
\begin{gathered}
{ }_{p} F_{q}\left(\begin{array}{c}
a_{1}, \ldots, a_{p} \\
a_{1}+m_{1}, \ldots, a_{n}+m_{n}, b_{n+1}, \ldots, b_{q}
\end{array} \mid z\right)=\prod_{j=1}^{n} \frac{\left(a_{j}\right)_{m_{j}}}{\left(m_{j}-1\right) !} \sum_{k=1}^{n} \sum_{j_{1}=0}^{m_{1}-1} \ldots \sum_{j_{n}=0}^{m_{n}-1} \frac{1}{a_{k}+j_{k}} \\
\prod_{l=1}^{n} \frac{\left(1-m_{l}\right)_{j_{l}}}{j_{l} !} \prod_{\substack{i=1 \\
i \neq k}}^{n} \frac{1}{a_{i}+j_{i}-a_{k}-j_{k}}{ }_{p-n+1} F_{q-n+1}\left(\begin{array}{c}
a_{k}+j_{k}, a_{n+1}, \ldots, a_{p} \\
a_{k}+j_{k}+1, b_{n+1}, \ldots, b_{q}
\end{array} \mid z\right), \\
m_{n} \in \mathbb{Z} \wedge m_{n}>0 \wedge n \leq q \wedge a_{i}+j_{i} \neq a_{k}+j_{k}, \forall j_{i}=0, \ldots \ldots m_{i}, 1 \leq i, j \leq n .
\end{gathered}
$$

This can be used to reduce the ${ }_{d} F_{d}$ of the form in Eqn. (22) to (i) a sum over ${ }_{1} F_{1}$ in odd dimensions, (ii) a sum over ${ }_{3} F_{3}$ in even dimensions $d>2$. In $d=2{ }_{d} F_{d}$ is simply ${ }_{2} F_{2}$ which can be examined directly. We demonstrate these results explicitly in appendix C.

Specifically, in odd dimensions there is a sum over ${ }_{1} F_{1}\left(\begin{array}{c}a_{k}+j_{k} \\ a_{k}+j_{k}+1\end{array} \mid-N\right)$, which using

$$
\begin{aligned}
{ }_{1} F_{1}\left(\begin{array}{c}
a \\
a+1
\end{array} \mid-z\right) & =a(z)^{-a}(\Gamma(a)-\Gamma(a, z)) \\
\Gamma(a, z) & \propto e^{-z} z^{a-1}\left(\frac{(2-a)(1-a)}{z^{2}}-\frac{1-a}{z}+\ldots+1\right) / ;(|z| \rightarrow \infty) .
\end{aligned}
$$

gives a leading order dependence of $N^{-a_{k}-j_{k}}$, for the smallest values of $a_{k}+j_{k}$ which is $k=1$, $j_{1}=0$, which makes it $N^{-\frac{2}{d}-m}$. In even dimensions for $d>2$, the dependence on $N$ appears in a 
sum over

$$
{ }_{3} F_{3}\left(\begin{array}{c}
\frac{2}{d} k+m+j_{k}, \frac{2}{d} l+m+1, m+1 \\
\frac{2}{d} k+m+j_{k}+1, \frac{2}{d} l+m+2, m+3
\end{array} \mid-N\right) .
$$

We obtain an asymptotic expansion of this using Mathematica and find that the leading order contribution is again $N^{-a_{k}-j_{k}}$ and hence comes from the $k=1, j_{1}=0$ term which makes it $\sim N^{-\frac{2}{d}-m}$. In $d=2$, the hypergeometric function in Eqn. 200 is simply ${ }_{2} F_{2}\left(\begin{array}{c}m+1, m+1 \\ m+3, m+3\end{array} \mid-N\right)$ whose leading order contribution is of the form $N^{-1-m} \log N$. Combining these we find that $\left\langle N_{m}^{d}\right\rangle \sim$ $N^{2-2 / d}$ to leading order for $d>2$ and $\left\langle N_{m}^{d}\right\rangle \sim N \log N$ for $d=2$.

What our detailed calculations show, moreover, is that in all dimensions the coefficient of the leading order term takes the simple form:

$$
\frac{1}{m !} \Gamma\left(\frac{2}{d}+m\right) \frac{\Gamma(d)}{\left(\frac{d}{2}-1\right)\left(\frac{d}{2}+1\right)_{d-2}}
$$

which implies Eqn. (21). The subleading contributions however vary from dimension to dimension as

$$
\left\langle N_{m}^{d}\right\rangle(N)=\frac{N^{2-\frac{2}{d}}}{m !} \Gamma\left(\frac{2}{d}+m\right) \frac{\Gamma(d)}{\left(\frac{d}{2}-1\right)\left(\frac{d}{2}+1\right)_{d-2}}+ \begin{cases}\mathcal{O}(N) & \text { for } d=3 \\ \mathcal{O}(N \log N) & \text { for } d=4 \\ \mathcal{O}\left(N^{2-\frac{4}{d}}\right) & \text { for } d>4\end{cases}
$$

for all $d>2$ and

$$
\left\langle N_{m}^{2}\right\rangle(N)=N \log N+\mathcal{O}(N),
$$

for $d=2$. We refer the reader to appendix $\mathrm{C}$ for the details of the calculation.

In particular we note that all contributions are slower than $N^{2}$ and that the convergence towards the limit happens polynomially, and hence is quite slow. In Fig. 5 we plot $\left\langle N_{m}^{d}\right\rangle(N)$ for $d=4$ for a range of $N$-values, as well as the asymptotic limit. The slow convergence makes it clear that it will not be possible to test this limit computationally. In Fig. 5 we plot the asymptotic limits for various $d$. Notably, for $d=2 S_{m}^{2}=1$ and therefore independent of $m$.

\section{Defining Local Regions in a Causal Set}

The main goal of this work is to show that $\left\langle N_{m}^{d}\right\rangle$ can be used as a definition of locality in a causal set which faithfully embeds into a continuum spacetime. Conversely it can be used as a test for manifoldlikeness as well as a continuum dimension estimator.

Consider an $N$-element causal set $C$ which faithfully embeds into an Alexandrov interval $I[p, q]$ in $d$-dimensional Minkowski spacetime at a given density $\rho$. As we have just shown above, if one considers the ensemble of causal sets obtained via a Poisson sprinkling into $I[p, q]$, then the average $\left\langle N_{m}^{d}\right\rangle$ has a characteristic behaviour with $m$. For large enough $\rho$, the interval abundances $N_{m}(C)$ for a single "typical" realisation will with high probability "track" $\left\langle N_{m}^{d}\right\rangle$, i.e., $N_{m}(C) \sim\left\langle N_{m}^{d}\right\rangle(N \pm \sqrt{N})$ for all $\mathrm{m}$. This is what we would expect from a Poisson distribution. As we will show in the following section, this expectation is confirmed by simulations. Simulations moreover show that the distribution of the $N_{m}^{d}$ for any given $m$ for an ensemble of causal sets obtained via a Poisson sprinkling into $I[p, q]$ is nearly Gaussian with a standard deviation of $\sim \sqrt{N}$. 


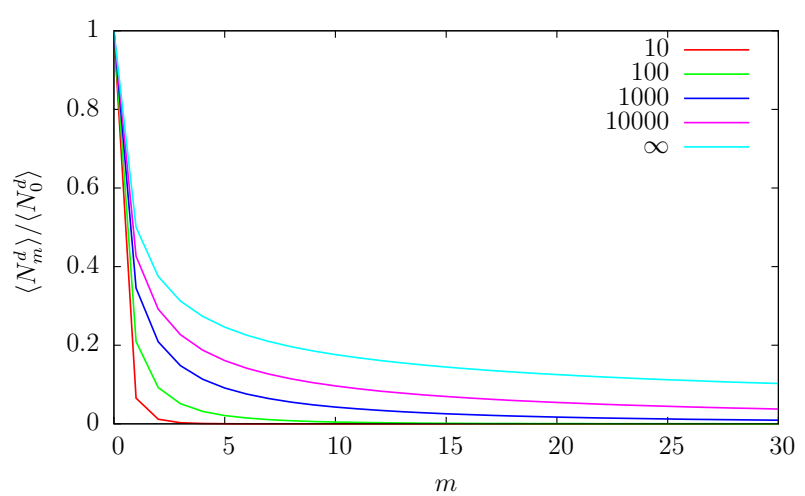

(a) Convergence in $4 d$

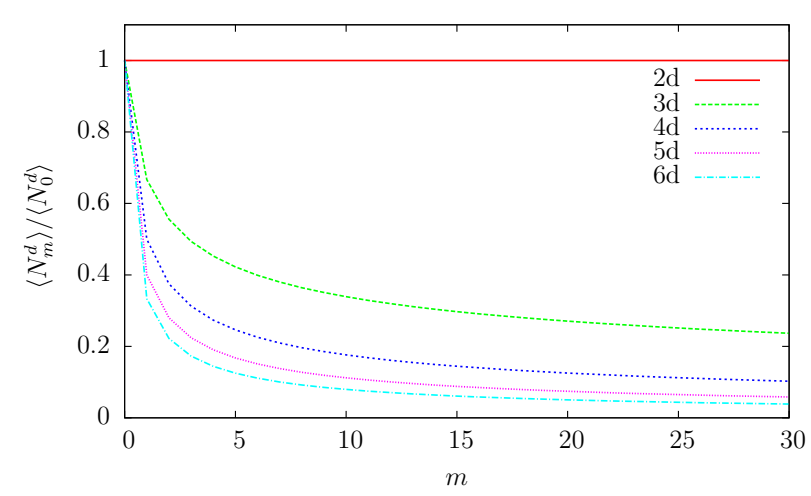

(b) Varying Dimension

Figure 5: These plots illustrate some properties of the $N \rightarrow \infty$ limit. The left hand plot shows the convergence of $\frac{\left\langle N_{m}^{d}\right\rangle}{\left\langle N_{0}^{d}\right\rangle}$ to $S_{m}^{d}$ in $4 \mathrm{~d}$ for $N=10,100,1000,10000$. The right hand plot shows how $S_{m}^{d}$ changes with dimension.

Importantly, the closeness of a typical $N_{m}(C)$ to $\left\langle N_{m}^{d}\right\rangle$ can be used as a characterisation of locality. Namely, if $C$ is such that $N_{m}(C) \sim\left\langle N_{m}^{d}\right\rangle(N \pm \sqrt{N})$ for all $m$, and for a fixed $d$ we will refer to it as a "local" causal set. For a causal set $\widetilde{C}$ which faithfully embeds into an Alexandrov interval $I[p, q]$ in an arbitrary curved $d$-dimensional spacetime, one expects that because of the deviation from flatness, $N_{m}(\widetilde{C})$ will differ significantly from the $\left\langle N_{m}^{d}\right\rangle$. Again, this is borne out by simulations. Thus, $\widetilde{C}$ is "non-local" in this sense. However, as long as the scale of flatness everywhere in $I[p, q]$ is much larger than the discreteness scale as discussed in Section 2 ] $\widetilde{C}$ will contain $N$ element sub-causal sets $C$ which lie in an approximately flat Alexandrov interval $I\left[p^{\prime}, q^{\prime}\right] \subset I[p, q]$. If $N$ is large enough, then $C$ will be local in the above sense. Thus, the $\left\langle N_{m}^{d}\right\rangle$ provide a strong characterisation of local regions in a causal set. Again, this is borne out by simulations on a class of curved spacetimes as well as those with non-trivial topology.

Thus, the function $\left\langle N_{m}^{d}\right\rangle$ suggests a criterion for "rigidity" of $C$ in the sense used by mathematicians. Namely, if $N_{m}(C) \sim\left\langle N_{m}^{d}\right\rangle(N \pm \sqrt{N})$ for all $m$, then it suggests that $C$ must faithfully embed into Minkowski spacetime of dimension $d$ at large enough embedding density. We now formalise these ideas as best as we can, leaving a more detailed study to future work.

Definition 1 We will say that an $N$-element causal set $C$ is strongly $d$-rigid if $\exists$ a $d$ for which $N_{m}(C) \sim\left\langle N_{m}^{d}\right\rangle(N \pm \sqrt{N})$. If $C$ possesses an $N^{\prime}$ element sub causal set $C^{\prime}$ which is strongly d-rigid, then $C$ is said to be weakly $d$-rigid with respect to $C^{\prime}$.

Clearly, strong $d$-rigidity is a necessary condition for an $N$-element causal set $C$ to faithfully embed into an Alexandrov interval of flat $d$-dimensional spacetime of volume $V$ as long as $N>>1$. On the other hand, weak $d$-rigidity is a rather weak necessary condition for $C$ to faithfully embed into a $d$-dimensional curved spacetime, since the only requirement is that there exist a local or strongly $d$-rigid sub-causal set $C^{\prime}$ in $C$. Indeed, in this case, one should expect a whole family of strongly $d$-rigid sub causal sets $\left\{C_{i}^{\prime}\right\}$ in $C$ for fixed $d$. However, a straightforward analysis of this case is far from clear at the moment and we leave this for future investigations.

\footnotetext{
${ }^{1}$ We will henceforth always assume that this condition is met.
} 
We summarise the above in the following Claim:

Claim 1 Let $C$ be an $N$-element causal set that faithfully embeds into an Alexandrov interval $I[p, q]$ in a d-dimensional spacetime such that the discreteness scale is much smaller than the scale of flatness everywhere. Then there exists a sub causal set $C^{\prime} \subset C$ of cardinality $N^{\prime}>>1$ such that $C^{\prime}$ is strongly d-rigid. Moreover, if $I[p, q]$ is an Alexandrov interval in d-dimensional Minkowski spacetime then for large enough $N, C$ is itself strongly d-rigid.

While the above arguments require that $N$ and $N^{\prime}$ be arbitrarily large in order to suppress fluctuations, the simulations that we will present in the next section show that the necessary condition works extremely well even for $N^{\prime}$ values as low as 100 for a single "typical" realisation of $C^{\prime}$. This is true both for the flat spacetime case as well as for regions where the scale of flatness is large . Of course, for a generic curved spacetime, one does need to go to higher densities, but here too, there is strong evidence that the numbers can be relatively small.

Could this condition also be sufficient for manifoldlikeness? As discussed above, in the general case, it clearly is insufficient since one needs requirements on an appropriately chosen family of strongly $d$-rigid sub-causal sets in $C$. On the other hand, it is a plausible sufficiency condition for a causal set to be faithfully embeddable into an interval in $d$-dimensional Minkowski spacetime. There are several hints that support this. We first note that the interval abundance profile for generic causal sets or Kleitman Rothschild posets [14] which dominate the class of posets for large $N$ differs vastly from $\left\langle N_{m}^{d}\right\rangle$. This difference in profile is easy to understand: these posets have a large number of links but almost no two or three element intervals. Thus, even at relatively small $m$, the interval abundances differ drastically from $\left\langle N_{m}^{d}\right\rangle$. Similar arguments can be made for the multiple layered class of causal sets studied in [15, 16] which are sub-dominant but are also largely devoid of small intervals with $m>1$. In Fig. 18 we show the interval abundances for a chain and a KR poset. Another example of a non manifoldlike causal set are the $2 D$ orders corresponding to the crystalline phase of [9]. These are again layered, much like the KR posets, but here too, there is a large deviation from the flat spacetime $\left\langle N_{m}^{d}\right\rangle$. Of course such examples cannot suffice since the space of causal sets is littered with those that have no simple characterisation. Hence we cannot at the moment prove that strong $d$-rigidity is violated for all causal sets which do not faithfully embed into an interval in flat spacetime.

On the other hand, as shown in Section 3.1 the ratio $\left\langle N_{m}^{d}\right\rangle /\left\langle N_{0}^{d}\right\rangle$ is scale invariant in the limit $N \rightarrow \infty$. In particular, this mimics the scale invariance of flat spacetime. Prompted by discussions with Sorkin we conjecture:

Conjecture 1 If the interval abundances $N_{m}(C)$ for an $N$-element causal set $C$ are such that that $N_{m}(C) \sim\left\langle N_{m}^{d}\right\rangle(N \pm \sqrt{N})$ for some $d$ in the large $N$ limit, then $C$ faithfully embeds into an Alexandrov interval in d-dimensional Minkowski spacetime.

In other words, we suggest that $\left\langle N_{m}^{d}\right\rangle$ provides a rigidity condition for a causal set to be approximated by an Alexandrov interval in Minkowski spacetime. A continuum version of this would require $C$ to moreover be scale invariant or homogeneous, and it would be interesting to explore whether there are examples of homogeneous orders like the Box spaces [17] which could provide counter-examples to the conjecture 2 .

\footnotetext{
${ }^{2}$ We thank Rafael Sorkin for discussions on the continuum limit.
} 


\section{Simulations}

We now show evidence for the above results and conjectures using simulations of relatively small causal sets. We consider causal sets that are sprinkled into flat and curved spacetime as well as non manifoldlike causal sets, using the Cactus-Code causal set framework [18, 19]. In particular, we perform our test on causal sets discretisations of flat spacetime for $d=2, \ldots, 4$ as well as on the 2-d cut-trousers and the flat geometries on $S^{1} \times \mathbb{R}$ and $T^{2} \times \mathbb{R}$. As examples of curved spacetime we consider FRW spacetimes for $d=4$ including deSitter spacetime, both for small and large scales of flatness, and find significant deviations from the flat spacetime curves in the case of a small scale of flatness. All these examples provide ample support for Claim 1

1 even for relatively small $N$. Next, we consider simulations of causal sets generated by transitive percolation for the specific cases studied in [10] and show that they do not pass our test for manifoldlikeness. Finally, as support for our conjecture, we examine distinctly non-manifoldlike causal sets, a chain and the class of Kleitman-Rothschild causal sets and show that, as expected, they fail our test of manifoldlikeness.

Once the causal set $C$ is simulated, the interval abundances can be obtained within an appropriately chosen order interval $I[p, q] \subseteq C$. We employ two different procedures for this purpose. The first procedure is a test of locality of an entire causal set. Here we consider sprinklings into a large interval in flat spacetime and "cap" $C$ to the past and the future by adding a pair of extra elements $p, q$ so that $I[p, q]=C$. This allows us to measure the interval abundance for the entire causal set. It is especially useful when comparing the results from simulations into flat spacetime with the analytic plots. Thus, we do not look for local regions in a given causal set, but test for the locality of an entire causal set, or in the language of the previous section whether it is strongly $d$-rigid for some $d$.

The second procedure is for finding local regions in a causal set $C$ which may not itself be local.Here we pick out an element $p \in C$ and then examine the set of order intervals to which $p$ belongs. By comparing with the $\left\langle N_{m}^{d}\right\rangle$ curves, one can then identify which of these order intervals might serve as a local neighbourhood of $p$. This method has two hurdles we must overcome.

Firstly, we can not control the location of $p$ in the embedding spacetime. This makes it hard to find intervals that sample a specific feature of a spacetime, say a singularity in the cut-trousers topology of 5.2 or a point close to the origin of the FRW spacetime. A little control can be exerted using the fact that the current Cactus code uses a natural labelling of the causal set, namely if $p \prec q$ then the labels satisfy $l(p)<l(q)$. Thus, picking a point with a low/ high labelling allows us to choose the lower/upper area of the region we sprinkled in.

Secondly, the number of intervals that contain an element can be very large even for moderately sized causal sets. This can be ameliorated by only examining intervals within a certain size range.

Thus our second procedure will be to pick an appropriate element in the causal set and then examine all intervals, within a certain size range, that contain this element.

\subsection{Flat spacetime simulations}

We first consider the class of causal sets obtained via a Poisson sprinkling into flat spacetime intervals with $\langle N\rangle=10^{d}$ elements using the existing Cactus code and calculate $N_{m}^{d}$ for each realisation of a sprinkled causal set, for $d=2, \ldots, 4$. We consider 1000 realisations in each case and calculate the standard deviation for the interval abundances. We find a remarkable agreement with the analytic curve for $N_{m}^{d}$ as shown in Fig. 6, where we have also plotted the analytic curves 
for $N \pm \sqrt{N}$.

We also find that for single realisations of a sprinkled causal set, the distribution of $N_{m}^{d}$ lies well within these curves as shown in 7. This plot also shows that the abundance can be used as a continuum dimension estimator. For causal sets that are non-manifoldlike this will give a null result since the profile of $N_{m}(C)$ will not match that of the continuum for any $d$.

\subsection{Examining other topologies}

As the simplest generalisation of flat spacetime intervals, we consider causal sets that are sprinkled into flat geometries with non-trivial spatial topology. An example of this is a "cut-trousers" topology in 2-d, with two disjoint spatial intervals $I \cup I$ joining up to give a single spatial interval $I$, as depicted in Fig. 8 ,

For the plot in Fig. 9 we obtained 100 realisations of a 1000-element causal set. For each of these, we picked large intervals by choosing a minimal element $p$ and a maximal element $q$ such that $|I[p, q]|$ is the largest interval. Because of the nature of the topology we are considering, these intervals are "incomplete" if taken to be embedded in flat spacetime, as shown in Fig. 8. We find that this size fluctuates by $380.68 \pm 14.01$ and thus, we can average over the 100 realisations to obtain the expectation value of the interval abundances for 380 element causal sets. This is within the standard deviation or fluctuation $\sqrt{380} \approx 19.5$ expected. As shown in Fig. 9, the curves $\left\langle N_{m}\left(C^{\prime}\right)\right\rangle$ exhibit a clear deviation from flat spacetime.

We also test causal sets sprinkled into $d=2$ and $d=3$ flat spacetimes with toroidal spatial topologies, i.e., with $M \equiv \mathbb{R} \times S^{1}$ (the cylinder) and $M \equiv \mathbb{R} \times T^{2}$, respectively. For the large intervals in $d=2$, we generate 100 realisations of 100-element causal sets via sprinkling and for $d=3,100$ we generate 100 realisations of1000-element causal sets. The results are shown in figure 10.

For the small intervals we look at single realisations of 10000-element causal sets and examines intervals of size 100 in both cases. We take more points to obtain a higher density causal set, which allows us to find 100-element intervals that do not probe the topology. As expected, for both $d=2$ and $d=3$ the large intervals, which wrap around the compact spatial topology have a distribution of intervals which has large deviations from the flat spacetime curve, but most of the small intervals do not. That some of the small intervals probe the topology of the space-time is due to the non-locality of the causal set. There will always be some small intervals that are almost light like, and thus probe the topology of the torus. We illustrate both the "non-local" and the "local" intervals in Fig. 11.

\subsection{Curved spacetime: FRW and DeSitter Spacetimes}

Next, we consider causal sets which are sprinkled into 4-d $k=0$ FRW spacetime with metric

$$
\mathrm{d} s^{2}=-\mathrm{d} t^{2}+a(t)^{2}\left(\sum_{i=1}^{4}\left(d x^{i}\right)^{2}\right)
$$

where

$$
a(t)=a_{0} t^{q} \quad \text { with: } \quad q=\frac{2}{3(1+w)}
$$




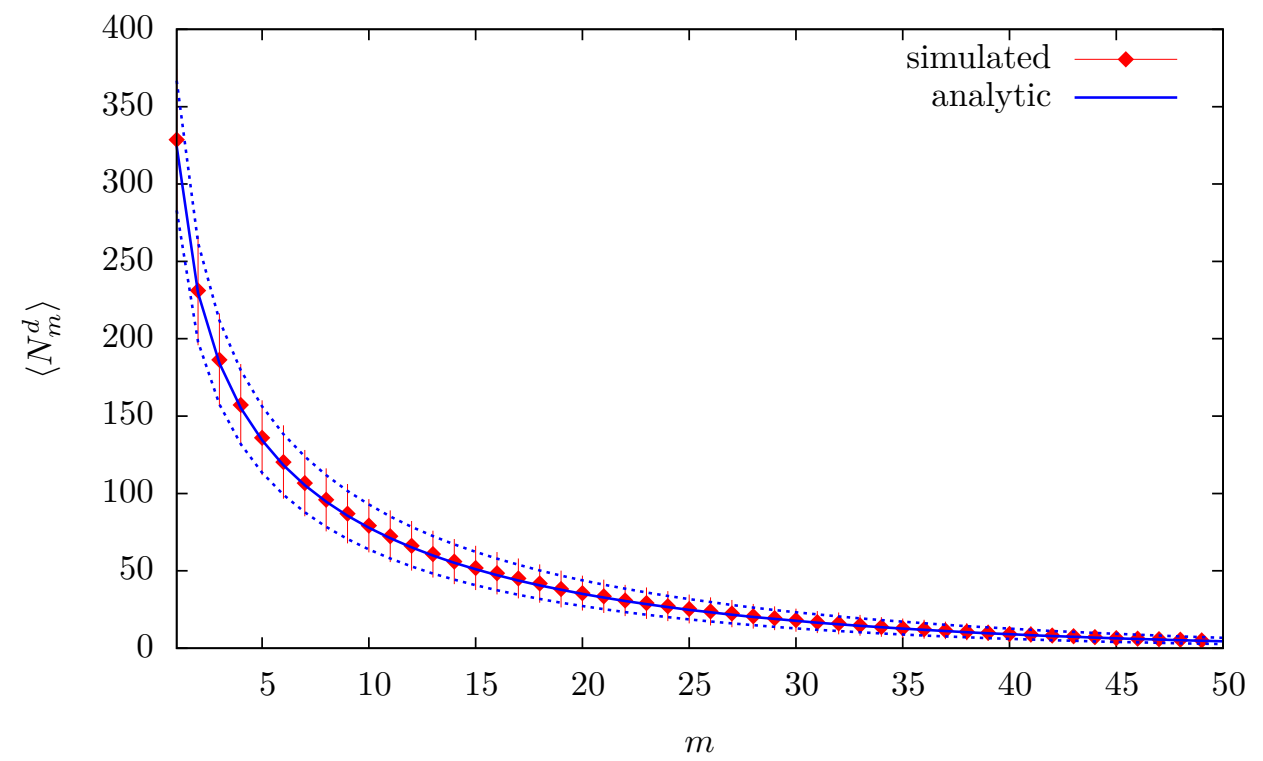

(a) 2d-100 Points

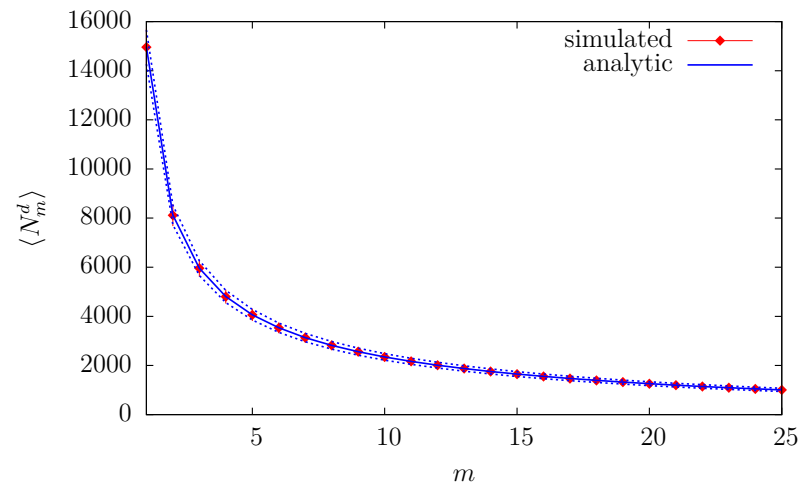

(b) 3d - 1000 Points

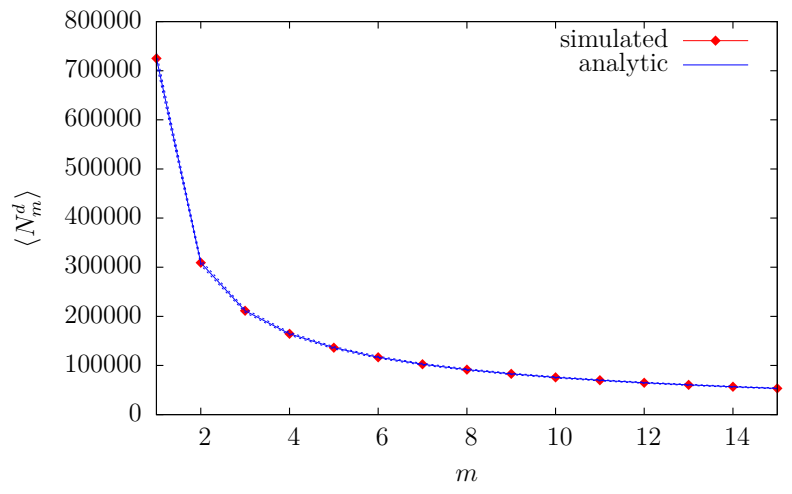

(c) 4d - 10000 Points

Figure 6: Simulations of the expectation value of interval abundances $\left\langle N_{m}(C)\right\rangle$ in flat space for $N=10^{d}$ element causal sets obtained by sprinkling 1000 times into an interval in flat spacetime. The red dots depict the simulations along with error bars. The solid blue line is $\left\langle N_{m}^{d}\right\rangle(N)$ while the dotted blue lines are $\left\langle N_{m}^{d}\right\rangle(N \pm \sqrt{N})$. 


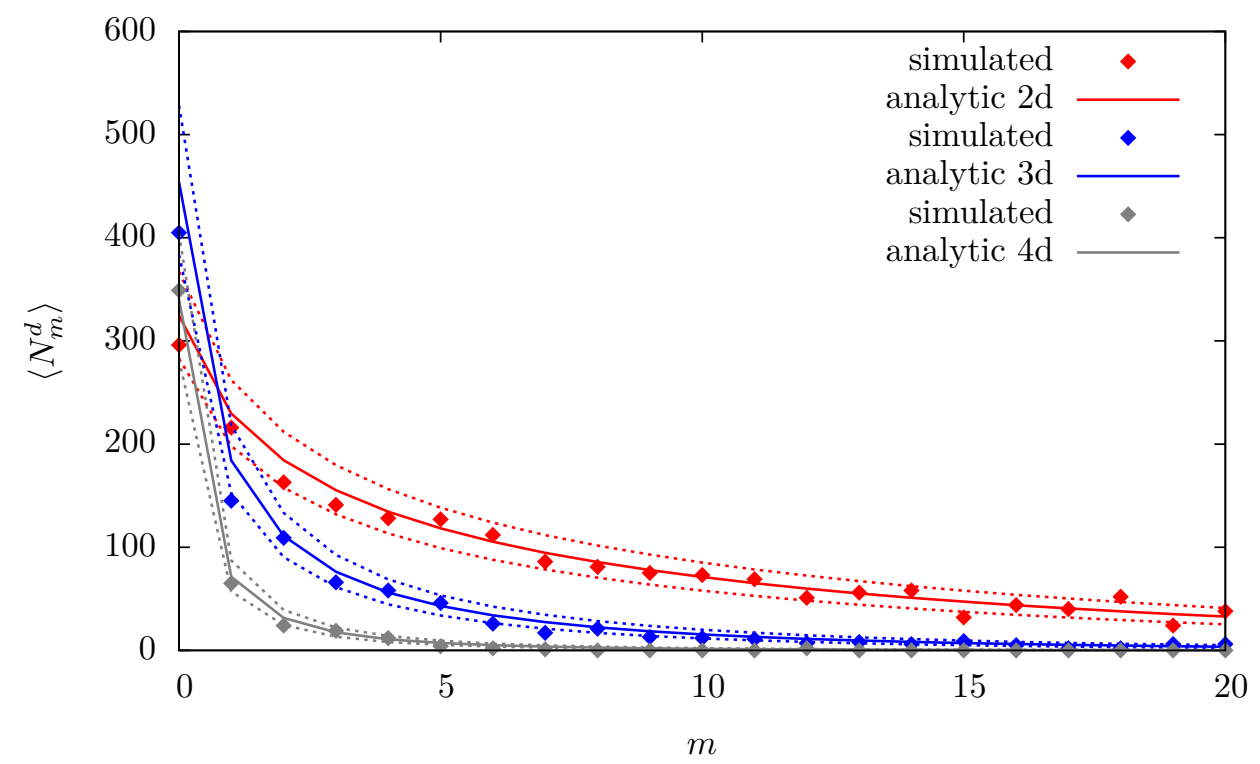

Figure 7: Simulations in flat space for single realisations of $N=100$ element causal sets obtained via Poisson sprinkling into flat spacetime intervals. The dots represent the simulations for a single causal set while the solid and dotted lines are $\left\langle N_{m}^{d}\right\rangle(N)$ and $\left\langle N_{m}^{d}\right\rangle(N \pm \sqrt{N})$, respectively. The agreement is striking.

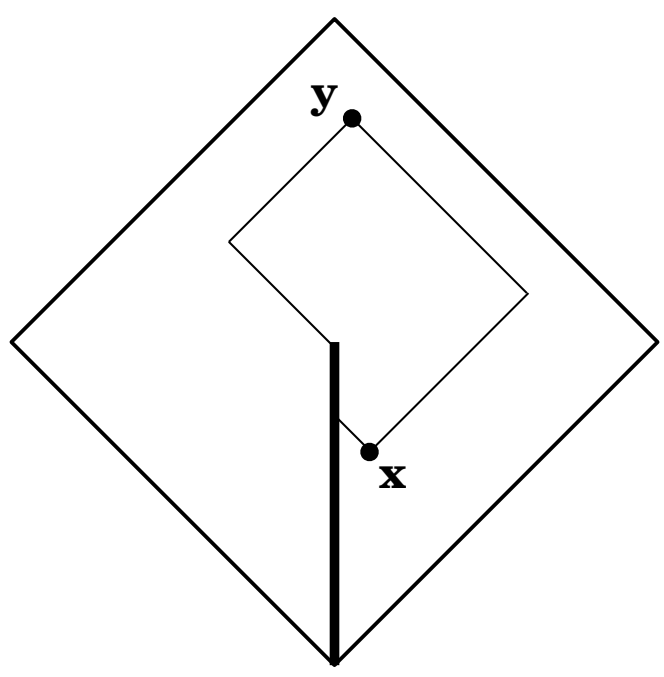

Figure 8: A sketch of the cut-trousers topology. The Alexandrov neighbourhood between the points $x$ and $y$ is modified by the cut. 


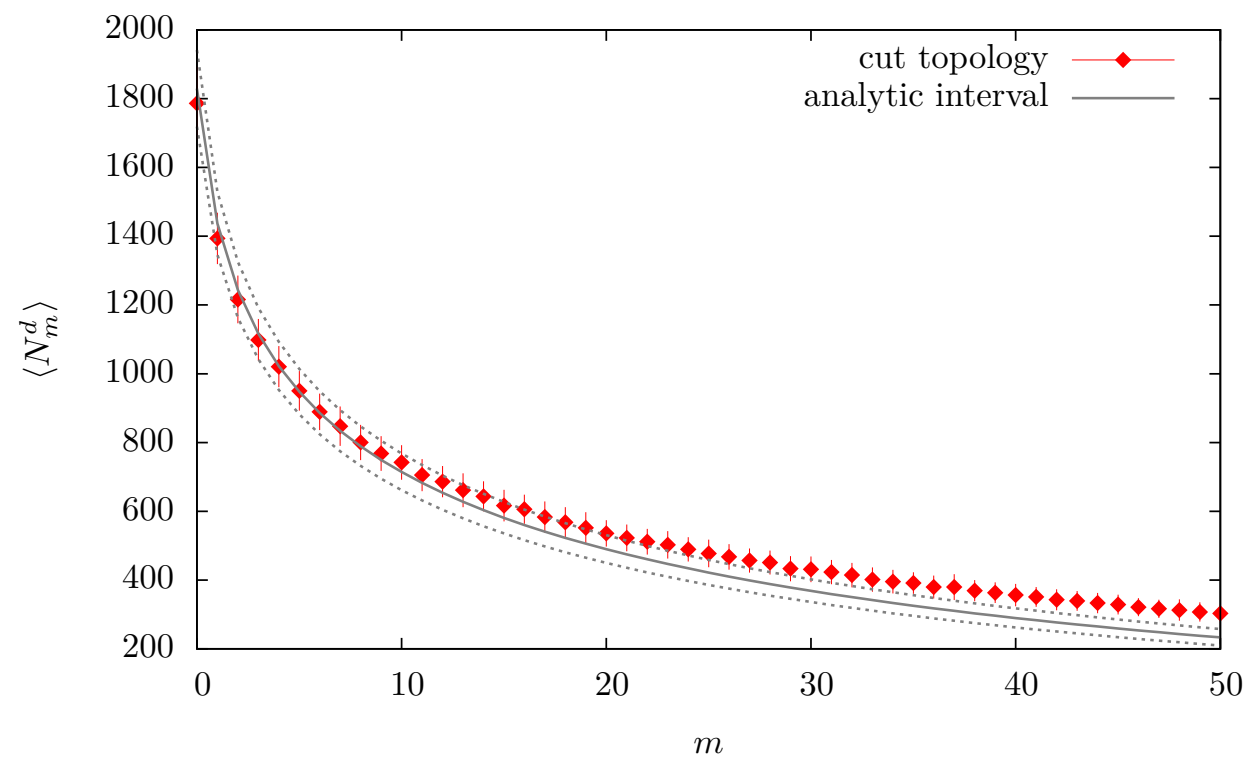

Figure 9: $\left\langle N_{m}(C)\right\rangle$ for the largest intervals contained in 100 realisations of $N=1000$ element causal sets obtained from sprinklings into the cut-trousers topology.

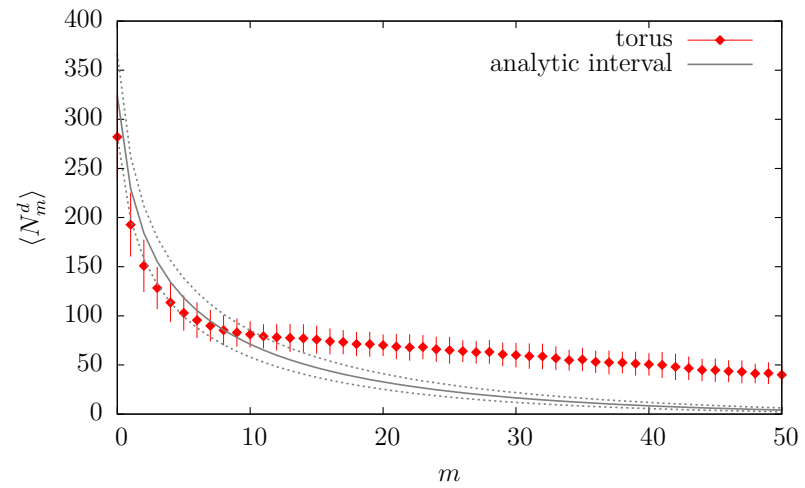

(a) 2-d 100 elements

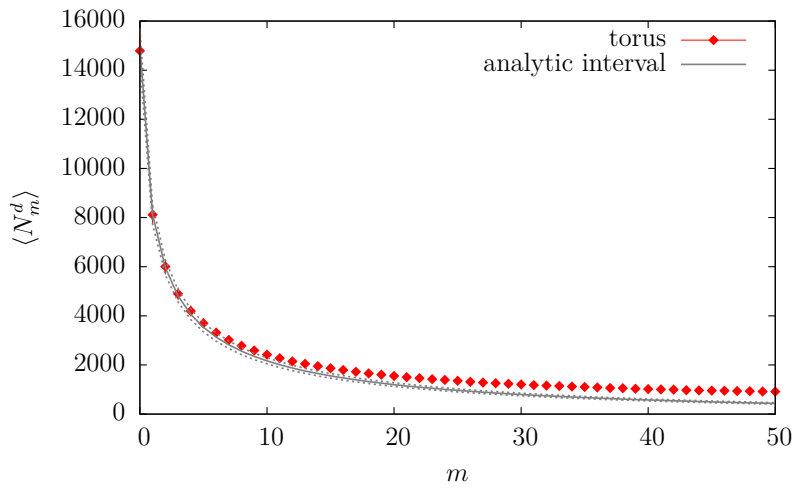

(b) 3-d 1000 elements

Figure 10: $\left\langle N_{m}(C)\right\rangle$ for large intervals in causal sets that are obtained from 100 sprinklings into flat spacetimes with toroidal spatial slices for $d=2,3$. 


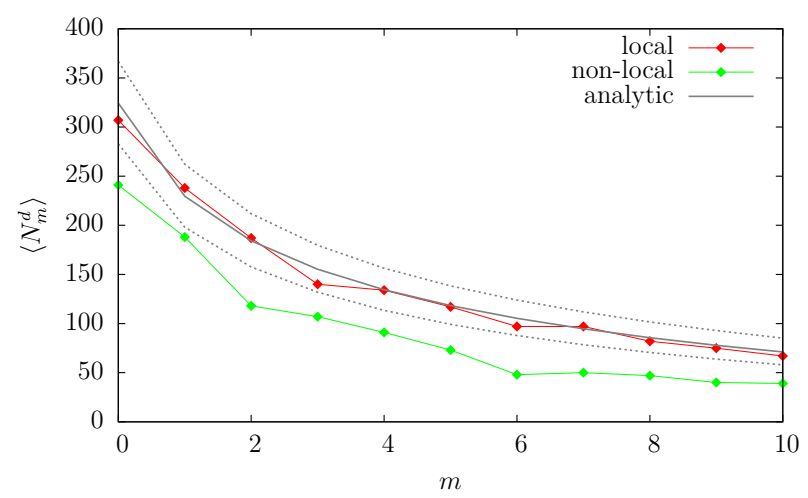

(a) 2-d local and non-local small intervals

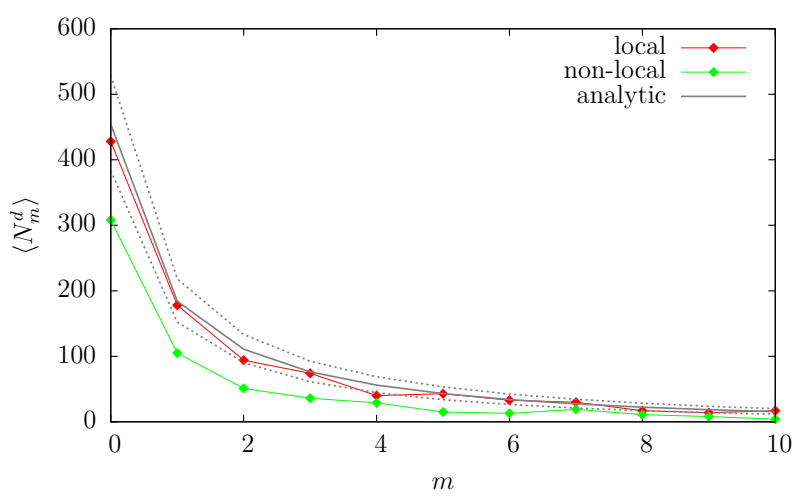

(b) 3-d local and non-local small intervals

Figure 11: $N_{m}\left(C^{\prime}\right)$ for a small 100-element interval causal set $C^{\prime}$ contained in a single 10000-element causal set obtained by sprinkling into flat spacetime with toroidal spatial slices in $d=2,3$.

with equation of state $p=w \rho$. We show our results from simulations for $w=0,1 / 3$ and the deSitter case $w=-1$ (resp. matter, radiation and cosmological constant dominated) as examples. In the deSitter case the deSitter radius arises as a new free parameter, we chose a radius of $\ell=1.3$. For each of these we examine 100 realisations of $\langle N\rangle=1000$-element causal sets and find the average interval abundances. We expect significant deviations from the flat spacetime case, due to the non-trivial spacetime curvature and our simulations do confirm this expectation.

We first show that the large intervals do not follow the flat spacetime characteristic curve. As for the intervals with non-trivial topology, the size of the intervals varies as $N_{x} \pm \sqrt{N}_{x}$. The results for all three choices clearly show the effect of curvature on the interval abundances.

To test manifoldlikeness of the causal set, we examine the intervals of size 100 containing a randomly chosen element in a single realisation of an 10000 element causal set. For $w=\frac{1}{3}$ and $w=0$ we found that there are intervals for which the abundances follow the flat spacetime curve and those which demonstrate significant deviations. As in the case of non-trivial topology, these latter interval neighbourhoods must sample a region in which the scale of flatness $\zeta^{-1}$ is small, i.e., they are elongated intervals. The result is shown in Fig. 13. The coloured boxes indicate the plotted intervals in co-moving coordinates, while the shading indicates the scale factor $a(t)$ which needs to be taken into consideration when comparing the intervals. Although the elongated intervals do not fit the curve for flat $4 \mathrm{~d}$ space, they are still not in agreement with higher or lower dimensional spacetime. If the same type of test is done on flat sprinkled causal sets, sprinkled sufficiently densely, there are no such stark differences between elongated and flat intervals. The same is true for intervals in deSitter spacetime. In figure (14) we show intervals of size 100 and 2000 picked out of a 10000 element deSitter sprinkling. They are as similar to each other as would be the case for flat space. This is because deSitter space is maximally symmetric. While the 100 intervals are in agreement with flat $4 \mathrm{~d}$ spacetime the 2000 element sets all show a significant deviation from flatness. 


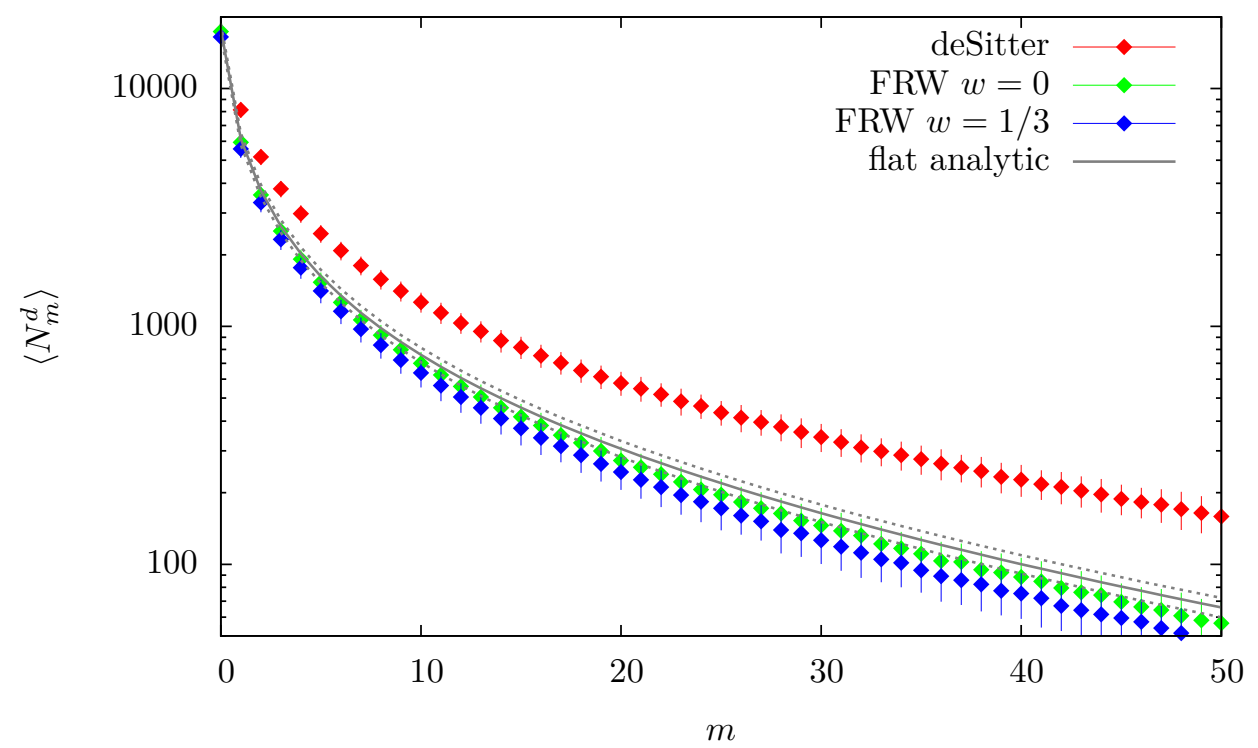

Figure 12: $\left\langle N_{m}(C)\right\rangle$ for $N=1000$ element causal sets obtained from sprinkling 100 times into $4 d$ FRW spacetimes which are $\Lambda$, matter or radiation dominated.

Table 1: Three sets of parameter values from [10] which we have examined.

\begin{tabular}{lllll}
\hline $\mathrm{p}$ & $\mathrm{N}$ & $\mathrm{d}$ & $\ell$ & $\mathrm{m}$ \\
\hline 0.03 & 1000 & $3 \mathrm{~d}$ & $2.331 \pm 0.011$ & $1.046 \pm 0.006$ \\
0.01 & 2000 & $3 \mathrm{~d}$ & $4.086 \pm 0.028$ & $1.136 \pm 0.006$ \\
0.005 & 15000 & $4 \mathrm{~d}$ & $6.20 \pm 0.12$ & $1.710 \pm 0.013$ \\
\hline
\end{tabular}

\subsection{Causal sets obtained from Transitive percolation}

Causal sets should ideally not be created by sprinkling but grow naturally from some form of process. One such process, put forward by Rideout and Sorkin is transitive percolation [20, 10].

In transitive percolation the causal set grows iteratively, one element at a time. Each element gets added and then connected to the older elements with a certain probability. The probability for an element at step $n$ to be connected to an element at step $n-1$ is denoted as $p$, which is the only free parameter. In [10, 20, causal sets of this type have been observed to have some manifoldlike characteristics. One thing that was examined was the functional relation between the proper time distance of two points and the volume that lies causally between them. It was found that for a variety of parameter combinations this curve can be well fit with the corresponding volume of a deSitter spacetime, using the deSitter radius $\ell$ and a proportionality factor between the length of the longest chain and the proper time $\tau$ as free parameters. To examine if percolated causal sets also appear manifoldlike under our new test we picked some of the possible parameter combinations, summarised in table 1 .

In the paper they fit the curve to only those intervals which for a given proper time, had the largest volume. We followed this up in finding those intervals and measuring their interval 

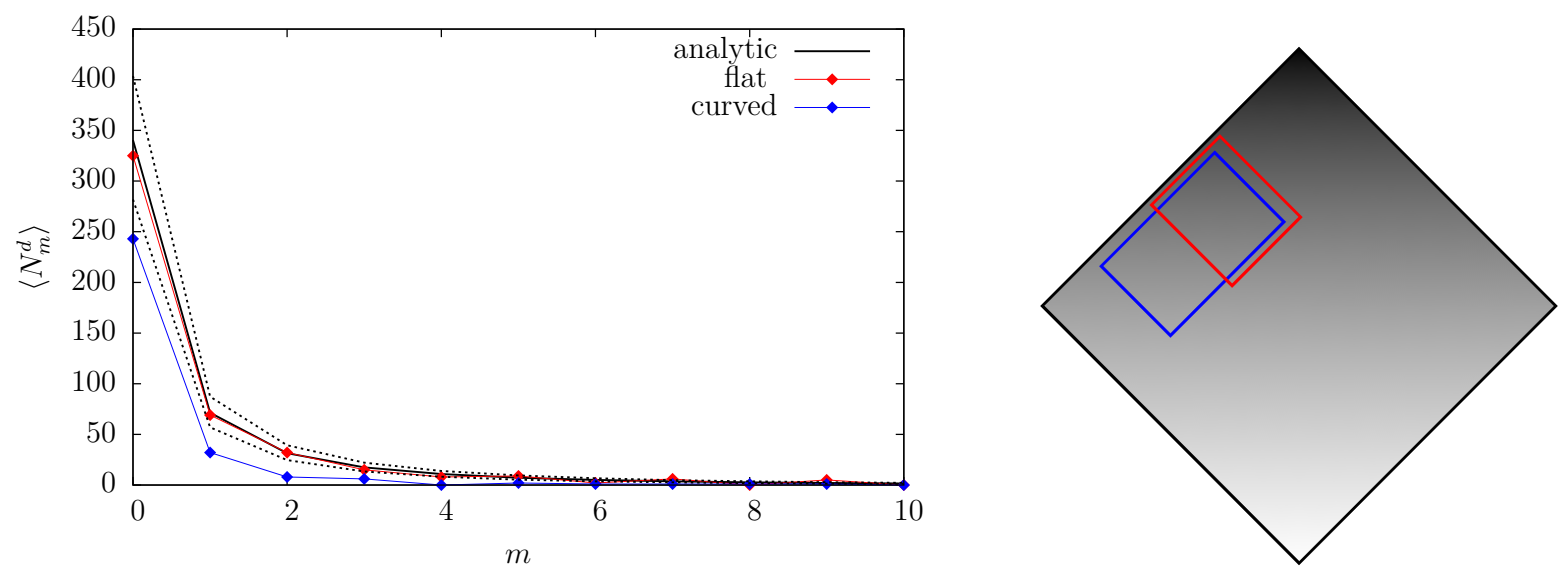

(a) FRW with $w=0$
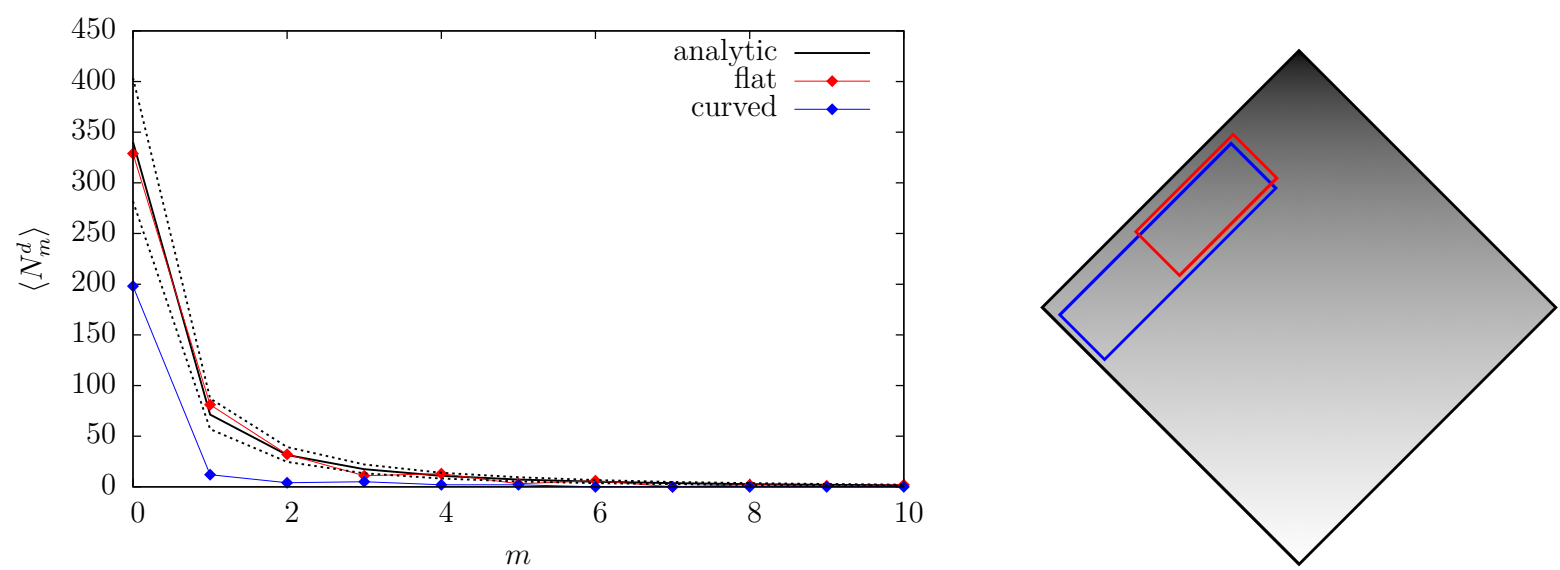

(b) FRW with $w=\frac{1}{3}$

Figure 13: Single realisations of small interval causal sets $C^{\prime}$ contained in an $N=10000$ element causal set $C$ obtained from a sprinkling into $4 d$ FRW spacetimes which matter or radiation dominated. The sketches on the right hand side show which intervals are local and which non-local, while the shading indicates the scale factor of the universe. 


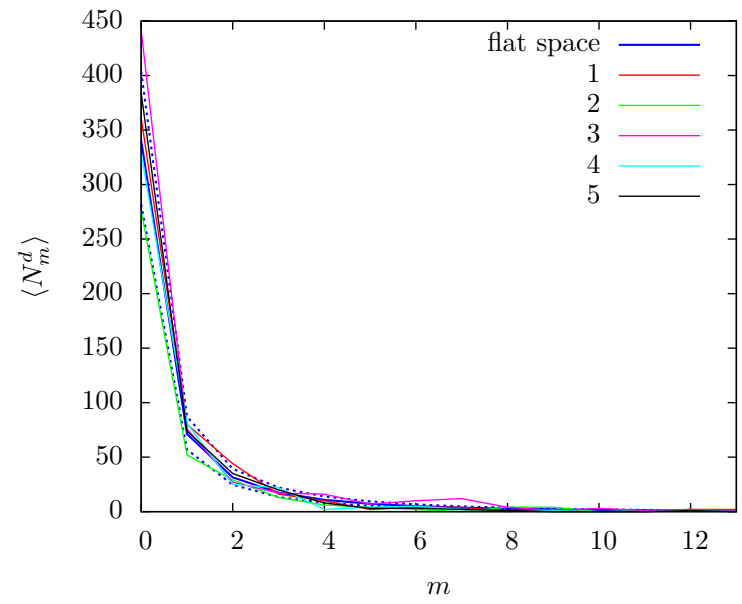

(a) 100 element deSitter intervals

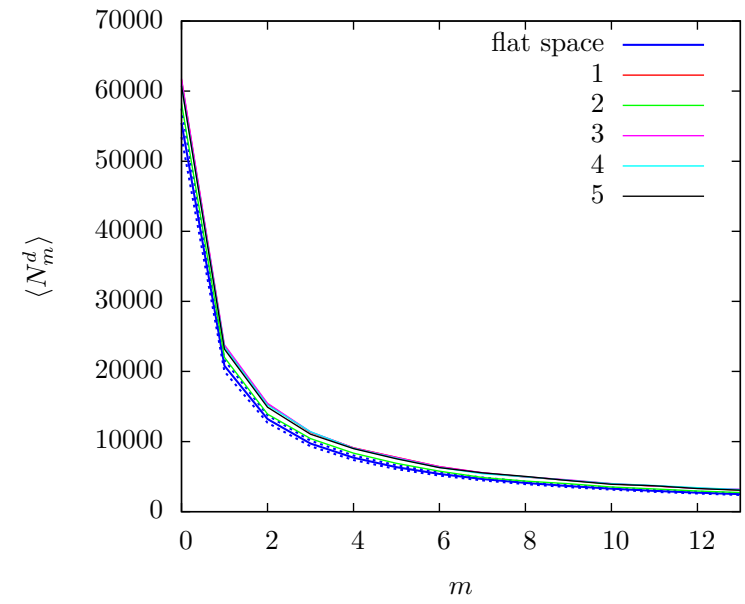

(b) 2000 element deSitter intervals

Figure 14: 5 single realisations of small interval causal sets $C^{\prime}$ contained in a $N=10000$ element causal set $C$ obtained from a sprinkling into $4 d$ deSitter spacetime.

abundances.

We created 100 percolated causal sets for each of the parameter combinations stated in table 1 and calculated the average interval abundances for intervals of different proper times. In Fig. 15 we plot this for the intervals of height 20. The behaviour of the interval abundance is similar for heights between around $10-50$ which is roughly the range of heights to which the deSitter volume profile was fitted in [10]. Indeed there is a striking dissimilarity with flat spacetime: the abundances $N_{m}\left(C^{\prime}\right)$ show a maximum at some $m>0$, unlike in flat spacetime for which $N_{m}\left(C^{\prime}\right)$ is a maximum for the links, i.e., $m=0$. This maximum shifts to larger $m$ as one examines larger Alexandrov intervals. One could perhaps argue that the different shape of the intervals could arise from curvature. However, the difference in shape persists even for very small intervals, which should look flat.This gives a strong indication that percolated causal sets are not manifoldlike.

Interestingly, while the interval abundance is clearly not that of flat spacetime it does converge towards the interval abundance of the dimension measured in [10] for large intervals. While the abundance of links and 1-element intervals for the percolated causal sets is very different from the analytic prediction, it falls off monotonically after the maximum and appears to get closer to the analytic prediction for manifoldlikeness.

This suggests that perhaps the percolated causal sets, while not manifoldlike in the small, might be manifoldlike at a coarse grained level and hence satisfy our test. The coarse graining procedure involves keeping each element of $C$ with a certain probability $P$. In Fig. 16 we show the interval abundance for coarse grained transitive percolated causal sets. We choose $P=0.25$, used the same values of $p$ as before, and fixed the number of elements such that it would agree with table 1 after coarse graining. Fig. 16 shows that coarse graining does change the structure of the interval abundances significantly, since the maximal abundance moves to smaller $m$ and the abundances become a monotonically decreasing function of $m$. However, despite this promising behaviour, the detailed curve differs strongly from the $\left\langle N_{m}^{d}\right\rangle$. Of course the size of the parameter space makes it hard to make a stronger claim, but for coarse-grainings where three-quarters, half, one quarter 


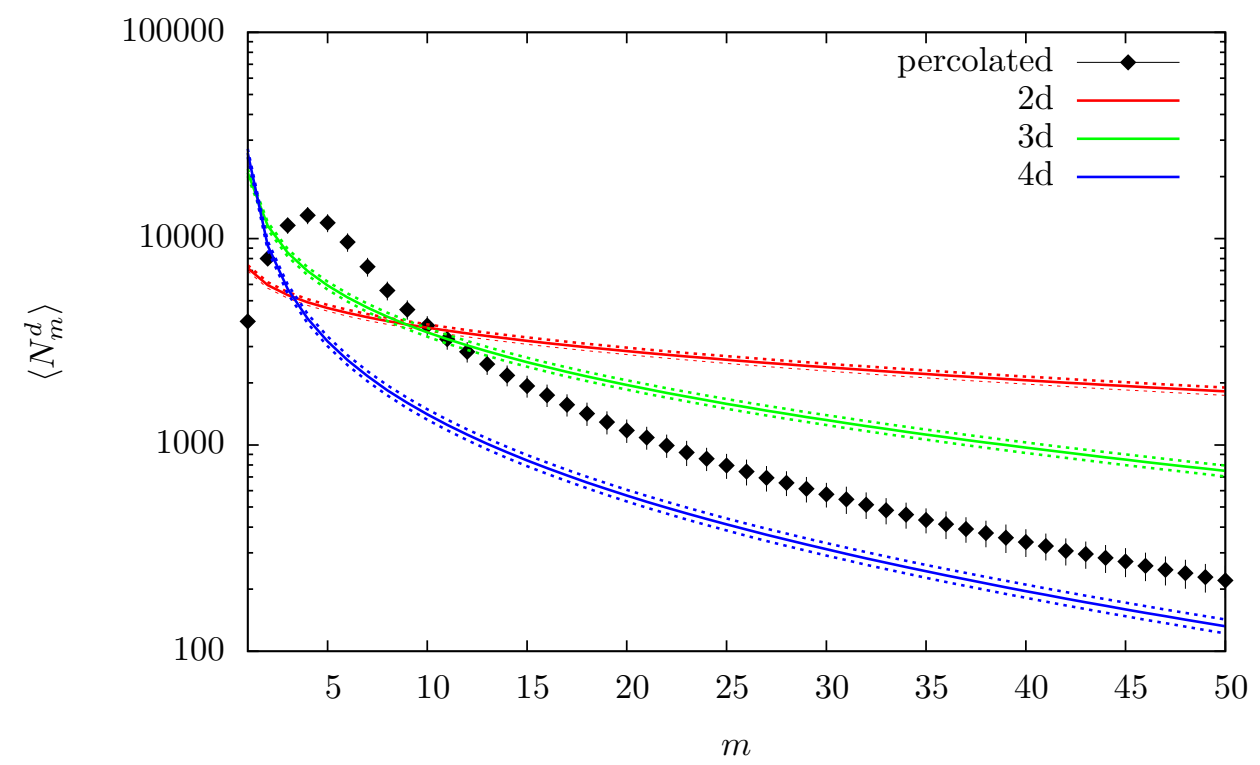

(a) $\mathrm{p}=0.005 \mathrm{~N}=15000$, average size $\langle N\rangle=1259.1 \pm 47.8$

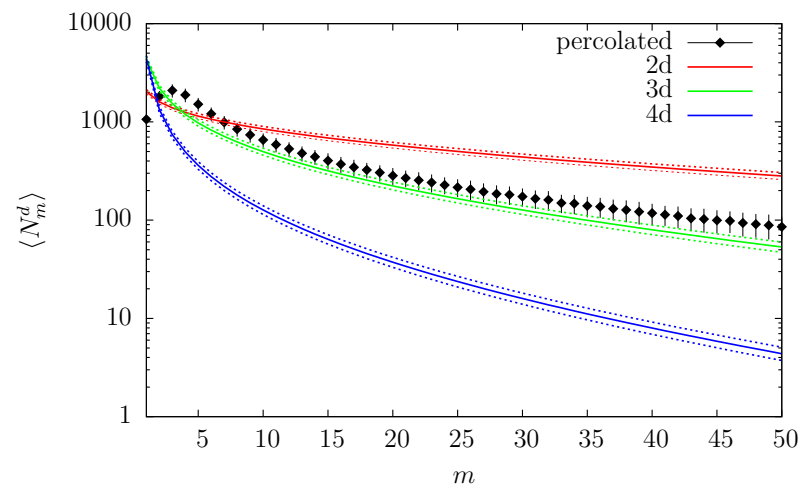

(b) $\mathrm{p}=0.03 \mathrm{~N}=1000$, average size $\langle N\rangle=335.2 \pm 25.9$

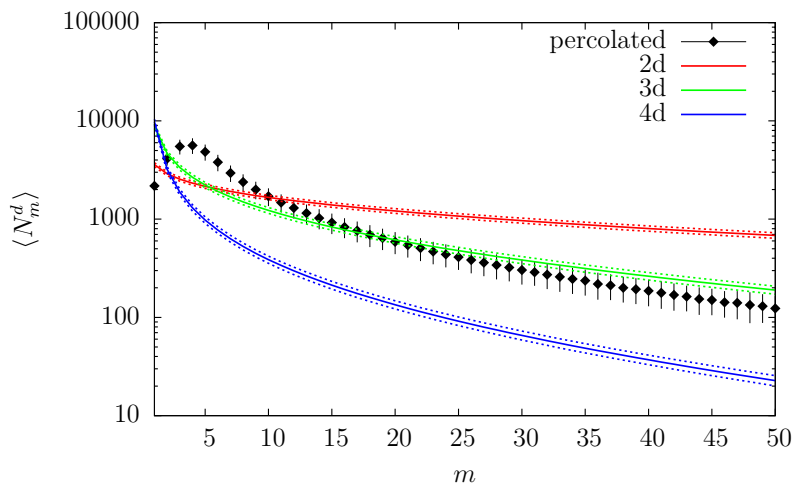

(c) $\mathrm{p}=0.01 \mathrm{~N}=2000$, average size $\langle N\rangle=695.7 \pm 53.5$

Figure 15: The $\left\langle N_{m}(C)\right\rangle$ for percolated causal sets whose longest chain has 20 elements, are shown in black and compared with the $\left\langle N_{m}^{d}\right\rangle$ for $d=2,3,4$. 


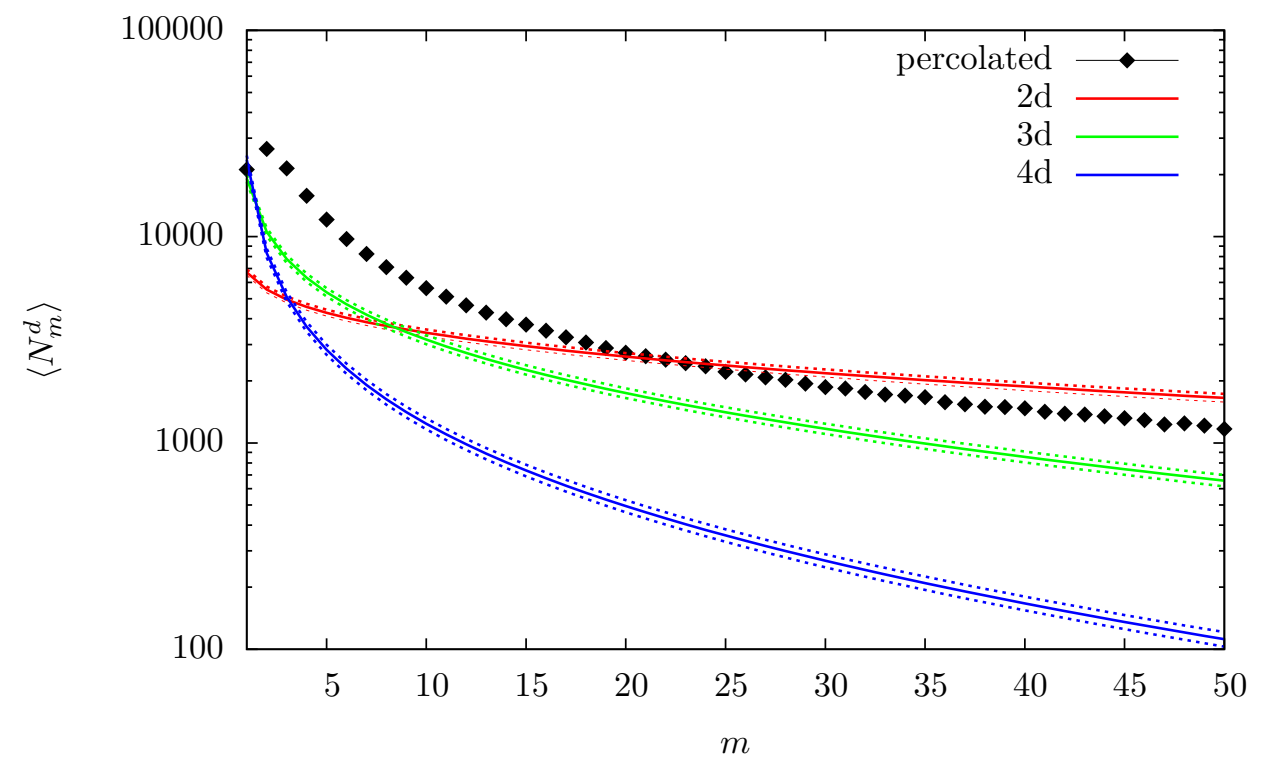

(a) $\mathrm{p}=0.005 \mathrm{~N}=15000$, average size $\langle N\rangle=1185.8 \pm 23.16$

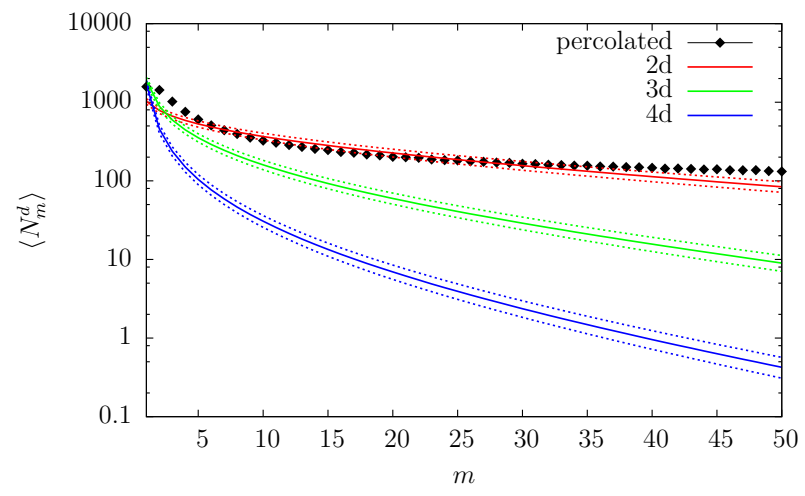

(b) $\mathrm{p}=0.03 \mathrm{~N}=1000$, average size $\langle N\rangle=249.5 \pm 11.7$

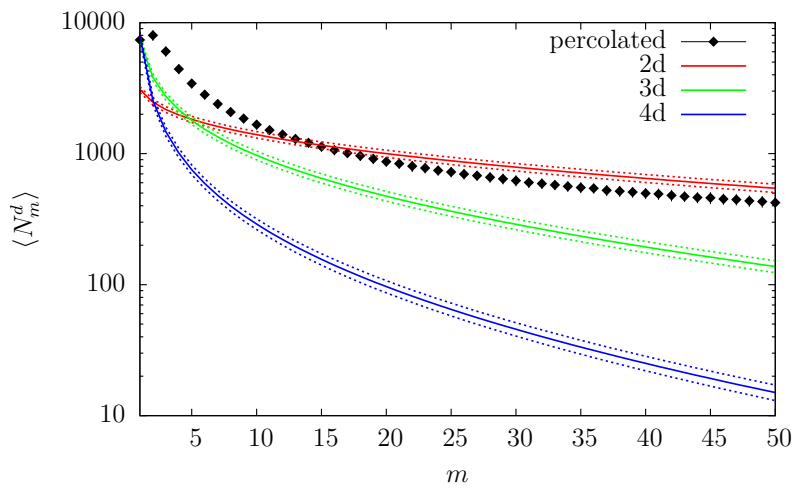

(c) $\mathrm{p}=0.01 \mathrm{~N}=2000$, average size $\langle N\rangle=615.5 \pm 30.3$

Figure 16: The $\left\langle N_{m}(C)\right\rangle$ for coarse grained percolated causal sets, whose longest chain is 20 elements long, is shown in black and compared with the $\left\langle N_{m}^{d}\right\rangle$ for $d=2,3,4$. 


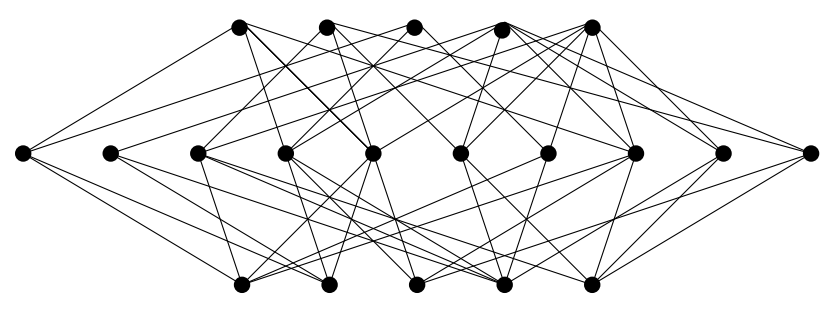

Figure 17: An example of a small KR- order

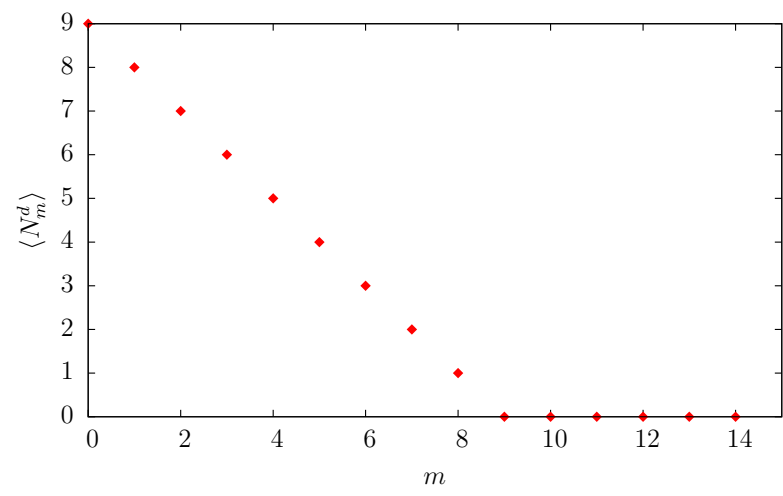

(a) chain

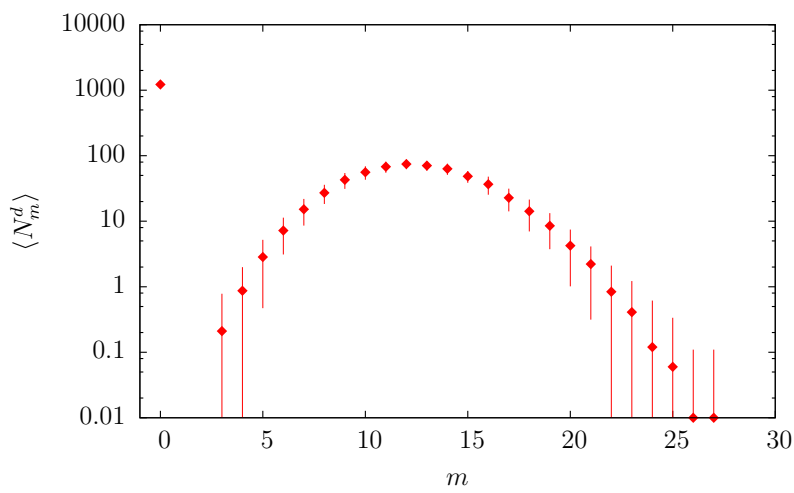

(b) KR-order

Figure 18: The $N_{m}(C)$ are plotted for two different non-manifoldlike causal sets. The left hand plot is for a chain of 10 elements and the right hand plot an average over 100 realisations of an 100-element KR-order.

or one tenth of the points were kept we did not find agreement with the analytic curve for flat spacetime. Further study to compare to the interval abundance for deSitter space might be useful, but first attempts at it do not indicate a substantial change in the results.

\subsection{Non manifoldlike causal sets}

There are several types of non-manifoldlike causal sets that can be examined in this manner. The first that come to mind is the totally ordered poset or chain and the totally unordered poset or antichain. The $N_{m}(C)$ for the former has a simple linearly decreasing behaviour with $m$ as depicted by the left hand plot of Fig. 18, while all the $N_{m}(C)$ for the latter are simply zero. However, apart from such exotic causal sets, one is interested in what $N_{m}(C)$ looks like for a more typical causal set. Here, we are aided by analytic results which tell us that as $N$ becomes large, the set of causal sets is dominated by those that are of the Kleitman-Rothschild or KR form. A sketch of a small KR- order is shown in Fig. 17. These are distinctly non-manifoldlike since they possess only three "moments of time". A typical KR orders has three layers with roughly $N / 4$ minimal and maximal elements each and $N / 2$-elements in the middle layer. Each minimal and maximal element are linked to roughly half the elements in the middle layer and every minimal element is related to every maximal element. Using David Rideout's Cactus thorn to generate KR orders, we perform 100 realisations of $N=100$ element KR orders to obtain $\left\langle N_{m}(C)\right\rangle$, which we show in Fig. 18 


\section{Conclusions and Outlook}

In this work we demonstrated that interval abundances $N_{m}(C)$ in a causal set $C$ provide an important class of observables for causal set theory. In particular, by comparing with the expectation value of the interval abundance $\left\langle N_{m}^{d}\right\rangle$ for an ensemble of causal sets obtained via a Poisson sprinkling into a flat spacetime interval, we showed that the $N_{m}(C)$ can be used to obtain "local" regions or sub-causal sets $C^{\prime} \subset C$ for a $C$ which faithfully embeds into a general curved spacetime. Conversely, the existence of local regions in a causal set is a necessary test for manifoldlikeness of $C$ and as a new continuum dimension estimator.

We began by obtaining closed form expressions for $\left\langle N_{m}^{d}\right\rangle$, and showed that the ratio $\left\langle N_{m}^{d}\right\rangle /\left\langle N_{0}^{d}\right\rangle$ is independent of the size $N$ of the causal set to leading order. This scale invariance reflects that of flat spacetime and suggests a rigidity condition encoded by this class of observables. This prompted us to conjecture that knowing the $N_{m}(C)$ for $N>>1$ is sufficient to determine if $C$ faithfully embeds into a flat spacetime interval of a given dimension. We tested these ideas with extensive simulations. We found that even for a relatively small ensemble of causal sets obtained via a Poisson sprinkling into an interval in Minkowski spacetime, the expectation value of the interval abundances matches very well with our analytic curves for $\left\langle N_{m}^{d}\right\rangle$. In addition the agreement is very good even for a single causal set, up to the expected Poisson fluctuations in the size of the causal set, $N \pm \sqrt{N}$, as shown in Fig. (7). This suggests a prescription for extracting the continuum spacetime dimension from a causal set and thus a necessary condition for it to be faithfully embeddable into Minkowski spacetime.

In curved spacetime we considered both FRW and deSitter spacetimes. The simulations agree with the $\left\langle N_{m}^{d}\right\rangle$ up to fluctuations as long as the scale of flatness is large, but deviate strongly from it when the scale of flatness is small. In the former case we found that the causal set represents a local or approximately flat spacetime region while in the latter case it is distinctly not local. We also examined the effect of topology on the $\left\langle N_{m}^{d}\right\rangle$ and found that again there is agreement with $\left\langle N_{m}^{d}\right\rangle$ only if the region explored in the spacetime is local.

We then examined a class of causal sets generated via transitive percolation to see if they passed our test for manifoldlikeness. In [10] it was claimed that a class of such causal sets possess manifoldlike properties and have a Myrheim-Myer manifold dimension of 3 or 4 . We tested several of these examples and found that they fail our test since the $N_{m}(C)$ do not agree with the $\left\langle N_{m}^{d}\right\rangle$ for any $d$. Hence we concluded that these causal sets are almost certainly not manifoldlike. However, it is possible that manifoldlikeness emerges after coarse graining. Preliminary tests showed that this is still not the case, but a more detailed study is currently underway [21].

Our simulations thus provide strong support for the rigidity conjecture in Section 4, namely that knowing $N_{m}(C)$ is sufficient to determine whether $C$ faithfully embeds into a flat spacetime interval or not. However, the question arises whether and why this class of observables is more special than others. For one, it does provide an entire class of observables, and this itself is useful. But this is also the case for the abundances of chains $\left\langle C_{m}\right\rangle$, where an $m$-chain is a totally ordered $m$-element subset of $C$. Thus, a similar analysis may be possible using the $\left\langle C_{m}\right\rangle$. However, what distinguishes $m$-intervals from $m$-chains is that the former do in fact encode Lorentz-invariant local information while this is not true of the latter. In particular, the set of $m$-element intervals with fixed future end point $q$ in a causal set that faithfully embeds into flat spacetime "layer" the past light cone of $q$ along its past invariant hyperbolae, for each $m$. For example, elements which are linked to $q$ lie within a volume $\sim \rho^{-1}$ to the past of $q$. Thus, as $m$ increases, one explores regions further and further from the past null cone boundary of $q$. However, a chain lacks the same local 
information. For example a relation or 2-chain $p<q$ could either be a link with $|I[p, q]|=0$ or separated by a very large interval size $|I[p, q]|>>1$. Thus, the number of relations to the future or the past of $p$ can lie arbitrarily far from the boundary of the light cones from $p$ - they are not nearest neighbours even in the Lorentzian sense. We believe it is this Lorentz-invariant locality of the $N_{m}(C)$ which make them useful in defining locality. There are no other obvious candidates for families of observables and it is therefore tempting to conclude that the $N_{m}(C)$ are unique in this sense.

It is relatively straightforward to extend these calculations to a region of small curvature using Riemann normal coordinates and the techniques of [22, 23. However, the expressions for the $\left\langle N_{m}^{d}\right\rangle$ are far more complex, and extracting even an analytic curve from them requires more computationally intensive tools than in the flat spacetime case. We leave such investigations to future work where effects of curvature can be studied in greater detail than in the present work.

Finally, local regions in a manifoldlike causal set $C$ could in principle be used to define a covering $\left\{C_{i}\right\}$ of $C=\cup_{i} C_{i}$, from which a nerve simplicial complex can be constructed. In the continuum, given a manifold $M$, a nerve simplicial complex can be obtained via a locally finite convex cover $\mathcal{O}=\left\{O_{i}\right\}, M=\cup_{i} O_{i}$, i.e., a cover in which (i) each set is convex, so that there exists a unique geodesic between any two points in the set (ii) every $x \in M$ is contained in a finite number of elements of $\mathcal{O}$. The nerve simplicial complex is obtained from $\mathcal{O}$ by mapping each $k$-wise intersection of sets in $\mathcal{O}$ to a $k$-simplex. This simplicial complex is then homotopic to $M$ as shown in [24]. In [25] a nerve simplicial complex was constructed to obtain the homology of spatial slices in both the continuum and in a causal set. One of the main obstructions to extending

it to the full continuum spacetime or the full causal set is that a locally finite cover built out of Alexandrov intervals does not have an obvious local characterisation. In particular, there is no way of distinguishing a convex from a non-convex Alexandrov interval purely order theoretically. Our prescription for locality in the discrete case however overcomes this difficulty and it would be interesting to see if the spacetime homology could in fact be recovered from such a local covering of a causal set.

Our work opens up several new arenas in the study of discrete causal structure, some of which may lead us closer to answering fundamental questions in causal set theory.

Acknowledgements: We thank Rafael Sorkin for discussions and David Rideout for help with the Cactus code, which we used extensively. The authors would like to thank the ICTP in Trieste for their kind hospitality during parts of this work, L.G. would also like to thank the Raman Research Institute in Bangalore for a very productive visit.

\section{Appendices}

\section{A A general formula for hypergeometric functions}

After solving the integration for the interval abundance in Section 3 it is necessary to find a closed form expression for sums of the type

$$
\sum_{n=0}^{\infty} \frac{(-\rho V)^{n}}{n !}\left(\prod_{i=1}^{q} \frac{1}{n+a_{i}}\right) \frac{\Gamma(x(n+c))}{\Gamma\left(x(n+c)+m_{1}\right)} \frac{\Gamma(x(n+e))}{\Gamma\left(x(n+e)+m_{2}\right)}
$$


In this expression it is assumed that $m_{1}, m_{2}$ are integers. We can then rewrite it as

$$
\sum_{n=0}^{\infty} \frac{(-\rho V)^{n}}{n !}\left(\prod_{i=1}^{q} \frac{1}{n+a_{i}}\right)\left(\prod_{k=0}^{m_{1}-1} \frac{1}{x(n+c)+k}\right)\left(\prod_{l=0}^{m_{2}-1} \frac{1}{x(n+e)+l}\right)
$$

To express this in a closed form we factorise out the $x$ and rewrite the products as gamma functions which gives

$$
\begin{array}{r}
\sum_{n=0}^{\infty} \frac{(-\rho V)^{n}}{n !} x^{m_{1}+m_{2}}\left(\prod_{i=1}^{q} \frac{\Gamma\left(n+a_{i}\right)}{\Gamma\left(n+a_{i}+1\right)}\right) \times \\
\left(\prod_{k=0}^{m_{1}-1} \frac{\Gamma\left(n+c+\frac{k}{x}\right)}{\Gamma\left(n+c+\frac{k}{x}+1\right)}\right)\left(\prod_{l=0}^{m_{2}-1} \frac{\Gamma\left(n+e+\frac{l}{x}\right)}{\Gamma\left(n+e+\frac{l}{x}+1\right)}\right)
\end{array}
$$

This can be rewritten using Pochhammer symbols $(a)_{n}=\Gamma(n+a) / \Gamma(a)$. Taking all the factors independent of $n$ out of the sum leads to

$$
\begin{gathered}
x^{m_{1}+m_{2}}\left(\prod_{i=1}^{q} \frac{\Gamma\left(a_{i}\right)}{\Gamma\left(a_{i}+1\right)}\right)\left(\prod_{k=0}^{m_{1}-1} \frac{\Gamma\left(c+\frac{k}{x}\right)}{\Gamma\left(c+\frac{k}{x}+1\right)}\right)\left(\prod_{l=0}^{m_{2}-1} \frac{\Gamma\left(e+\frac{l}{x}\right)}{\Gamma\left(e+\frac{l}{x}+1\right)}\right) \times \\
\sum_{n=0}^{\infty} \frac{(-\rho V)^{n}}{n !}\left(\prod_{i=1}^{q} \frac{\left(a_{i}\right)_{n}}{\left(a_{i}+1\right)_{n}}\right)\left(\prod_{k=0}^{m_{1}-1} \frac{\left(c+\frac{k}{x}\right)_{n}}{\left(c+\frac{k}{x}+1\right)_{n}}\right)\left(\prod_{l=0}^{m_{2}-1} \frac{\left(e+\frac{l}{x}\right)_{n}}{\left(e+\frac{l}{x}+1\right)_{n}}\right),
\end{gathered}
$$

which can then be reexpressed in terms of the $l+m_{1}+m_{2} F_{l+m_{1}+m_{2}}$ hypergeometric function

$$
\begin{aligned}
& \left(\prod_{i=1}^{q} \frac{1}{a_{i}}\right)\left(\prod_{k=0}^{m_{1}-1} \frac{1}{x c+k}\right)\left(\prod_{l=0}^{m_{2}-1} \frac{1}{x e+l}\right) \times \\
& l+m_{1}+m_{2} F_{l+m_{1}+m_{2}}\left(\begin{array}{c}
{\left[a_{i}\right],\left[c+\frac{k}{x}\right],\left[e+\frac{l}{x}\right]} \\
{\left[a_{i}+1\right],\left[c+\frac{k}{x}+1\right],\left[e+\frac{l}{x}+1\right]}
\end{array} \mid-\rho V\right),
\end{aligned}
$$

where the $\left[a_{i}\right]$ stand for $a_{1}, \ldots, a_{q}$ and $\left[c+\frac{k}{x}\right]$ (respective $\left[e+\frac{l}{x}\right]$ ) do stand for $m_{1}\left(m_{2}\right)$ terms in which $k(l)$ varies from 0 to $m_{1}-1$ ( 0 to $\left.m_{2}-1\right)$. One last simplification allows us to write

$$
\begin{array}{r}
\left(\prod_{i=1}^{q} \frac{1}{a_{i}}\right) \frac{\Gamma(x c) \Gamma(x e)}{\Gamma\left(x c+m_{1}\right) \Gamma\left(x e+m_{2}\right)} \times \\
l+m_{1}+m_{2} F_{l+m_{1}+m_{2}}\left(\begin{array}{c}
{\left[a_{i}\right],\left[c+\frac{k}{x}\right],\left[e+\frac{l}{x}\right]} \\
{\left[a_{i}+1\right],\left[c+\frac{k}{x}+1\right],\left[e+\frac{l}{x}+1\right]}
\end{array} \mid-\rho V\right)
\end{array}
$$

If $c, e, a_{i}$ have an integer distance smaller than $m_{1}\left(m_{2}\right)$ it is possible to simplify this further since arguments of the hypergeometric function that arise on both sides cancel each other. In our calculation these simplifications will indeed take place but details are specific to each case. 


\section{B Deriving the $m \neq 0$ case from the $m=0$ case using Hypergeo- metric function identities}

We can derive $\left\langle N_{m}^{d}\right\rangle$ from $\left\langle N_{0}^{d}\right\rangle$ by taking derivatives. The expression for $N_{0}^{d}$ is of the form

$$
\chi(\rho V)^{2}{ }_{p} F_{p}\left(\begin{array}{c}
a_{1}, \ldots, a_{p} \\
b_{1}, \ldots, b_{p}
\end{array} \mid-\rho V\right),
$$

where we have lumped some of the dimension dependent constants into the term $\chi$. Using (17) the expression for the $\left\langle N_{m}^{d}\right\rangle$ is

$$
\chi \frac{(-\rho)^{m+2}}{m !} \frac{\partial^{m}}{\partial \rho^{m}} V_{p}^{2} F_{p}\left(\begin{array}{c}
a_{1}, \ldots, a_{p} \\
b_{1}, \ldots, b_{p}
\end{array} \mid-\rho V\right) .
$$

We use the identity [26]

$$
\left.\frac{\partial^{m}}{\partial z^{m}}{ }_{p} F_{p}\left(\begin{array}{c}
a_{1}, \ldots, a_{p} \\
b_{1}, \ldots, b_{p}
\end{array}\right) z\right)=\frac{\prod_{j=1}^{p}\left(a_{j}\right)_{m}}{\prod_{j=1}^{p}\left(b_{j}\right)_{m}} F_{p}\left(\begin{array}{c}
m+a_{1}, \ldots, m+a_{p} \\
m+b_{1}, \ldots, m+b_{q}
\end{array} \mid z\right)
$$

to simplify Eqn. (39) to

$$
\chi \frac{(\rho V)^{m+2}}{m !} \frac{\prod_{j=1}^{p}\left(a_{j}\right)_{m}}{\prod_{j=1}^{p}\left(b_{j}\right)_{m}} F_{p}\left(\begin{array}{c}
a_{1}+m, \ldots, a_{p}+m \\
b_{1}+m, \ldots, b_{p}+m
\end{array} \mid-\rho V\right)
$$

This expression allows for further simplifications, depending on the $a_{j}, b_{j}$. These can be done for each individual case.

\section{Derivation of the Continuum Limit}

The quantity we calculate is

$$
S_{m}^{d} \equiv \lim _{\rho \rightarrow \infty} \frac{\left\langle N_{m}^{d}\right\rangle(\rho, V)}{\left\langle N_{0}^{d}\right\rangle(\rho, V)},
$$

where $\left\langle N_{m}^{d}\right\rangle$ is given by Eqn. 201. To investigate the $N \rightarrow \infty$ limit of the $\left\langle N_{m}^{d}\right\rangle$ we need a large $N=\rho V$ expansion of the hypergeometric functions that appear in Eqn. 20, which when appropriately rearranged are of the form

$$
{ }_{d} F_{d}\left(\begin{array}{c}
a_{1}, \ldots, a_{d} \\
a_{1}+2, \ldots, a_{d}+2
\end{array} \mid-N\right), \quad a_{i}=\frac{2 i}{d}+m, i=1, \ldots d-1, \quad a_{d}=1+m .
$$

We make repeated use of the identity Eqn. (23) as well as the identity Eqn. (24). Since the first identity can not be used if two of the $a_{i}$ are equal, or equal up to an integer whose absolute value is smaller than $m_{i}$, we will need to be careful in even dimensions. For $d=2$, in particular we need a different approach. We will thus treat odd and even dimensions separately and $d=2$ as a separate case. 


\section{C.1 Odd dimensions:}

This involves the most straightforward application of Eqn. (23) to expand (43):

$$
\begin{aligned}
& { }_{d} F_{d}\left(\begin{array}{c}
a_{1}, \ldots, a_{d} \\
a_{1}+2, \ldots, a_{d}+2
\end{array} \mid-z\right)=\prod_{j=1}^{d}\left(a_{j}\right)_{2} \sum_{k=1}^{d} \sum_{j_{1}=0}^{1} \ldots \sum_{j_{d}=0}^{1} \frac{1}{a_{k}+j_{k}} \\
& \prod_{l=1}^{d}(-1)^{j_{l}} \prod_{\substack{i=1 \\
i \neq k}}^{d} \frac{1}{a_{i}+j_{i}-a_{k}-j_{k}}{ }_{1} F_{1}\left(\begin{array}{c}
a_{k}+j_{k} \\
a_{k}+j_{k}+1
\end{array} \mid-z\right)
\end{aligned}
$$

For us $m_{l}=2 j_{l}=0,1$ so that $\left(1-m_{l}\right)_{j_{l}}=(-1)^{j_{l}}$. Using Eqn. (24) in 44).

$$
=\sum_{k=1}^{d} \sum_{j_{1}=0}^{1} \ldots \sum_{j_{d}=0}^{1}(z)^{-a_{k}-j_{k}}\left(\Gamma\left(a_{k}+j_{k}\right)-\Gamma\left(a_{k}+j_{k}, z\right)\right) \prod_{l=1}^{d}\left(a_{l}\right)_{2}(-1)^{j_{l}} \prod_{\substack{i=1 \\ i \neq k}}^{d} \frac{1}{a_{i}+j_{i}-a_{k}-j_{k}} .
$$

For $z \rightarrow \infty$ the terms containing $\Gamma(a, z)$ fall off like $e^{-z} z^{a-1}$, c.f. Eqn. (25) and do not contribute in the large $z$ limit. Thus, to leading order the hypergeometric function is a power series with terms $z^{-a_{i}-j_{i}}$. The leading order term is therefore $a_{1}=\frac{2}{d}+m, j_{1}=0$, while the next to leading order is $a_{d}=1+m$ for $d=3$ and $a_{2}=\frac{4}{d}+m$ for $d \geq 5$. We then only need to calculate the case $k=1, j_{k}=0$.

Combining the products in Eqn. (45), we then sum over the $j_{i}$,

$$
\sum_{j_{i}=0}^{1} \frac{\left(a_{i}\right)_{2}(-1)^{j_{i}}}{a_{i}+j_{i}-a_{1}}=\left(a_{i}\right)_{2}\left(\frac{1}{a_{i}-a_{1}}-\frac{1}{a_{i}+1-a_{1}}\right)=\frac{\left(a_{i}\right)_{2}}{\left(a_{i}-a_{1}\right)\left(a_{i}+1-a_{1}\right)}
$$

after which we take the product over $i$ to obtain

$$
\left(a_{k}\right)_{2} \prod_{\substack{i=1 \\ i \neq k}}^{d} \frac{\left(a_{i}\right)_{2}}{\left(a_{i}-a_{k}\right)\left(a_{i}+1-a_{k}\right)}=\left(\frac{2}{d}+m\right)_{2} \frac{(m+1)_{2}}{\left(1-\frac{2}{d}\right)\left(2-\frac{2}{d}\right)} \prod_{i=2}^{d-1} \frac{\left(\frac{2}{d} i+1\right)_{2}}{\frac{2}{d}(i-1)\left(\frac{2}{d}(i-1)+1\right)}
$$

We do the product for the different parts separately:

$$
\begin{aligned}
& \left(\frac{2}{d}+m\right)_{2} \prod_{i=2}^{d-1}\left(\frac{2}{d} i+1\right)_{2}=\prod_{i=1}^{d-1}\left(\frac{2}{d} i+m\right)\left(\frac{2}{d} i+m+1\right) \\
& =\left(\frac{2}{d}\right)^{2 d-2}\left(\frac{d}{2} m+1\right)_{d-1}\left(\frac{d}{2}(m+1)+1\right)_{d-1} \\
& \prod_{i=2}^{d-1} \frac{1}{\frac{2}{d}(i-1)+1} \frac{d}{2(i-1)}=\left(\frac{2}{d}\right)^{-2 d+2} \frac{1}{\Gamma(d-1)\left(\frac{d}{2}-1\right)_{d-2}}
\end{aligned}
$$

These are combined to find

$$
z^{-\frac{2}{d}-m} \frac{2 \Gamma\left(\frac{2}{d}+m\right)(m+1)(m+2)}{\Gamma(d)(d-2))\left(\frac{d}{2}+1\right)_{d-2}}\left(\frac{d}{2}(m+1)+1\right)_{d-1}\left(\frac{d}{2} m+1\right)_{d-1}+ \begin{cases}\mathcal{O}\left(z^{-m-1}\right) & \text { if } d=3 \\ \mathcal{O}\left(z^{-\frac{4}{d}-m}\right) & \text { if } d \geq 5\end{cases}
$$


Inserting this into Eqn. (20) for large $N$ gives

$$
N_{m}^{d}(N)=\frac{N^{2-\frac{2}{d}}}{m !} \Gamma\left(\frac{2}{d}+m\right) \frac{\Gamma(d)}{\left(\frac{d}{2}-1\right)\left(\frac{d}{2}+1\right)_{d-2}}+ \begin{cases}\mathcal{O}(N) & \text { if } d=3 \\ \mathcal{O}\left(N^{2-\frac{4}{d}}\right) & \text { if } d \geq 5\end{cases}
$$

which gives us Eqn. (21).

\section{C.2 Even dimensions:}

In even dimensions it is possible for two of the $a_{i}$ to be equal, or equal up to an integer whose absolute value is less than $m_{i}$. This therefore requires more care. The arguments of the hypergeometric functions in Eqn. (20) however do admit a non-degenerate split:

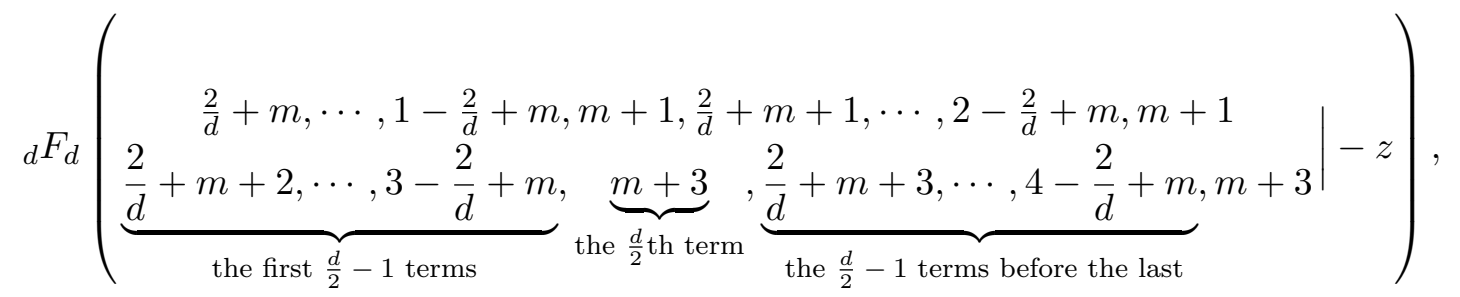

which can be shuffled to simplify the calculation. Exchanging the first $\frac{d}{2}-1$ terms and the $\frac{d}{2}-1$ terms before the last in the upper row, changes the relationship between the top and bottom row. Instead of a hypergeometric function of the form (43) we now have one of the form

$$
{ }_{d} F_{d}\left(\begin{array}{c}
a_{1}, \cdots, a_{\frac{d}{2}-1}, a_{\frac{d}{2}}, a_{\frac{d}{2}+1}, \cdots, a_{d-1}, a_{d} \\
a_{1}+1, \cdots, a_{\frac{d}{2}-1}+1, a_{\frac{d}{2}}+2, a_{\frac{d}{2}+1}+3, \cdots, a_{d-1}+3, a_{d}+2
\end{array} \mid-z\right)
$$

We now proceed in two steps. The first is to use (23) on the first $\frac{d}{2}-1$ terms. Here $n=d / 2-1$, $m_{i}=1 \forall i \in[1, \ldots, d / 2-1]$ and hence $j_{i}=0 \forall i$. Thus

$$
\begin{aligned}
& { }_{d} F_{d}\left(\begin{array}{c}
\frac{2}{d}+m+1, \cdots, 2-\frac{2}{d}+m, m+1, \frac{2}{d}+m, \cdots, 1-\frac{2}{d}+m, m+1 \\
\frac{2}{d}+m+2, \cdots, 3-\frac{2}{d}+m, m+3, \frac{2}{d}+m+3, \cdots, 4-\frac{2}{d}+m, m+3
\end{array} \mid-z\right)= \\
& \sum_{\alpha=1}^{\frac{d}{2}-1} \frac{d}{2}+2 F_{\frac{d}{2}+2}\left(\begin{array}{c}
\frac{2}{d} \alpha+m+1, \frac{2}{d}+m, \cdots, 1-\frac{2}{d}+m, m+1, m+1 \\
\frac{2}{d} \alpha+m+2, \frac{2}{d}+m+3, \cdots, 4-\frac{2}{d}+m, m+3, m+3
\end{array} \mid-z\right) \prod_{\substack{j=1 \\
j \neq \alpha}}^{\frac{d}{2}-1} \frac{j+\frac{d}{2}(m+1)}{j-\alpha}
\end{aligned}
$$


Next we apply eqn. (23) for a second time, with $i=2, \ldots d / 2+1$. Now $n=d / 2$ and $m_{i}=3$ for $i \in[2, \ldots, d / 2]$ while $m_{d / 2+1}=2$. Thus,

$$
\begin{aligned}
& \frac{d}{2}+2 F_{\frac{d}{2}+2}\left(\begin{array}{c}
\frac{2}{d} \alpha+m+1, \frac{2}{d}+m, \cdots, 1-\frac{2}{d}+m, m+1, m+1 \\
\frac{2}{d} \alpha+m+2, \frac{2}{d}+m+3, \cdots, 4-\frac{2}{d}+m, m+3, m+3
\end{array} \mid-z\right)= \\
& \left(\prod_{j=1}^{\frac{d}{2}} \frac{\left(\frac{2}{d} j+m\right)_{3}}{2}\right) \cdot \frac{2}{m+3} \sum_{k=1}^{\frac{d}{2}} \sum_{j_{1}}^{2} \cdots \sum_{j_{\frac{d}{2}-1}}^{2} \sum_{j_{\frac{d}{2}}}^{1} \frac{(-1)^{j_{d}}}{\frac{2}{d} k+j_{k}+m}\left(\prod_{l=1}^{\frac{d}{2}-1} \frac{(-2)_{j_{l}}}{j_{l} !}\right) \times \\
& \left(\prod_{\substack{i=1 \\
i \neq k}}^{\frac{d}{2}} \frac{1}{\frac{2}{d}(i-k)+j_{i}-j_{k}}\right){ }_{3} F_{3}\left(\begin{array}{c}
\frac{2}{d} k+m+j_{k}, \frac{2}{d} \alpha+m+1, m+1 \\
\frac{2}{d} k+m+j_{k}+1, \frac{2}{d} \alpha+m+2, m+3
\end{array} \mid-z\right)
\end{aligned}
$$

To take the limit $z \rightarrow \infty$ we need to expand this ${ }_{3} F_{3}$ for large $z$. We cannot do the entire expansion because of the special cases where $\frac{2}{d} k+m+j_{k}=\frac{2}{d} \alpha+m+1$ and $\frac{2}{d} k+m+j_{k}=m+1$. We thus need to make the expansion for three different possibilities:

- $\frac{2}{d} k+m+j_{k} \neq \frac{2}{d} \alpha+m+1 \neq m+1$ in this case we can find an exact expansion for all $z$

- $\frac{2}{d} k+m+j_{k}=\frac{2}{d} \alpha+m+1$ for this case we can take an expansion in large $z$ and find that it's leading order contributions in that limit are $z^{-1-m} \log z$.

- $\frac{2}{d} k+m+j_{k}=m+1$ we can again take a large $z$ expansion and find terms proportional to $z^{-m-1} \log z$ in leading order. (23)

The expansions for the first case, $\frac{2}{d} k+m+j_{k} \neq \frac{2}{d} \alpha+m+1 \neq m+1$, can be found using Eqn.

$$
\begin{aligned}
& { }_{3} F_{3}\left(\begin{array}{c}
\frac{2}{d} k+m+j_{k}, \frac{2}{d} \alpha+m+1, m+1 \\
\frac{2}{d} k+m+j_{k}+1, \frac{2}{d} \alpha+m+2, m+3
\end{array} \mid-z\right)= \\
& e^{-z} \frac{(2+m)\left(\frac{2}{d} \alpha+m+1\right)\left(\frac{2}{d} k+m+j_{k}\right)}{\frac{2}{d} \alpha\left(\frac{2}{d} k+j_{k}-1\right)} \\
& -\frac{(1+m)(2+m)\left(\frac{2}{d} \alpha+m+1\right)\left(\frac{2}{d} k+m+j_{k}\right)}{\left(\frac{2}{d} \alpha-1\right)\left(\frac{2}{d} k+j_{k}-2\right)} z^{-2-m}(\Gamma(2+m)-\Gamma(2+m, z)) \\
& +\frac{(2+m)\left(\frac{2}{d} \alpha+m+1\right)\left(\frac{2}{d} k+m+j_{k}\right)}{\frac{2}{d} \alpha\left(\frac{2}{d} k+j_{k}-1\right)} z^{-1-m}(\Gamma(2+m)-\Gamma(2+m, z)) \\
& -\frac{(2+m)(1+m)\left(\frac{2}{d} \alpha+m+1\right)\left(\frac{2}{d} k+m+j_{k}\right)}{\frac{2}{d} \alpha\left(\frac{2}{d} \alpha-1\right)\left(\frac{2}{d}(\alpha-k)+1-j_{k}\right)} z^{-1-m-\frac{2}{d} \alpha}\left(\Gamma\left(\frac{2}{d} \alpha+1+m\right)-\Gamma\left(\frac{2}{d} \alpha+1+m, z\right)\right) \\
& -\frac{(2+m)(1+m)\left(\frac{2}{d} \alpha+m+1\right)\left(\frac{2}{d} k+m+j_{k}\right)}{\left(\frac{2}{d} k+j_{k}-2\right)\left(\frac{2}{d} k+j_{k}-1\right)\left(\frac{2}{d}(k-\alpha)+j_{k}-1\right)} z^{-j_{k}-m-\frac{2}{d} k}\left(\Gamma\left(j_{k}+m+\frac{2}{d} k\right)-\Gamma\left(j_{k}+m+\frac{2}{d} k, z\right)\right)
\end{aligned}
$$


For the second and third case we use Mathematica to obtain the expansion. In the second case, $\frac{2}{d} k+$ $m+j_{k}=\frac{2}{d} \alpha+m+1$,

$$
\begin{aligned}
& { }_{3} F_{3}\left(\begin{array}{c}
\frac{2}{d} \alpha+m+1, \frac{2}{d} \alpha+m+1, m+1 \\
\frac{2}{d} \alpha+m+2, \frac{2}{d} \alpha+m+2, m+3
\end{array} \mid-z\right)= \\
& \frac{(m+2)\left(\frac{2}{d} \alpha+m+1\right)^{2}}{\left(\frac{2}{d} \alpha\right)^{2}} z^{-m-1} \Gamma(m+2)-\frac{(m+1)\left(\frac{2}{d} \alpha+m+1\right)^{2}}{\left(\frac{2}{d} \alpha-1\right)^{2}} z^{-m-2} \Gamma(m+3) \\
& +\frac{(m+1)(m+2)\left(\frac{2}{d} \alpha+m+1\right)}{\left(\frac{2}{d} \alpha-1\right) \frac{2}{d} \alpha} \Gamma\left(m+\frac{2}{d} \alpha+2\right) z^{-\frac{2}{d} \alpha-m-2}\left(\frac{2 \frac{2}{d} \alpha-1}{\left(\frac{2}{d} \alpha-1\right) \frac{2}{d} \alpha}+\psi^{(0)}\left(m+\frac{2}{d} \alpha+1\right)+\log (z)\right) \\
& +\cdots
\end{aligned}
$$

and in the third case, $\frac{2}{d} k+m+j_{k}=m+1$,

$$
\begin{aligned}
& { }_{3} F_{3}\left(\begin{array}{l}
m+1, \frac{2}{d} \alpha+m+1, m+1 \\
m+2, \frac{2}{d} \alpha+m+2, m+3
\end{array} \mid-z\right)= \\
& -\frac{(1+m)^{2}(2+m)\left(1+m+\frac{2}{d} \alpha\right)}{\left(\frac{2}{d} \alpha\right)^{2}\left(\frac{2}{d} \alpha-1\right)} z^{-1-m-\frac{2}{d} \alpha} \Gamma\left(1+m+\frac{2}{d} \alpha\right) \\
& -\frac{(m+1)\left(1+m+\frac{2}{d} \alpha\right)}{\left(\frac{2}{d} \alpha\right)^{2}} z^{-1-m} \Gamma(3+m)\left(2-\log z+\Psi^{(0)}(m+1)\right)+\cdots
\end{aligned}
$$

The leading order terms are $z^{-\frac{2}{d}-m}$, for $k=1, j_{k}=0$ from Eqn. 55 . The next to leading order term is $z^{-\frac{4}{d}-m}$ if $d>4$, while for $d=4$ it is $z^{-m-1} \log z$, from (56) and (57). Thus to leading order we find

$$
\begin{aligned}
& \left(\prod_{j=1}^{\frac{d}{2}} \frac{\left(\frac{2}{d} j+m\right)_{3}}{2}\right) \cdot \frac{2}{m+3} \sum_{j_{1}}^{2} \cdots \sum_{j_{\frac{d}{2}-1}}^{2} \sum_{j_{\frac{d}{2}}}^{1} \frac{1}{\frac{2}{d} 1+m}\left(\prod_{l=1}^{\frac{d}{2}-1} \frac{(-2)_{j_{l}}}{j_{l} !} \prod_{i=2}^{\frac{d}{2}-1} \frac{1}{\frac{2}{d}(i-1)+j_{i}}\right) \times \\
& \frac{(-1)^{j_{\frac{d}{2}}}}{1+j_{\frac{d}{2}}-\frac{2}{d}} \frac{(2+m)(1+m)\left(\frac{2}{d} \alpha+m+1\right)\left(\frac{2}{d}+m\right)}{\left(\frac{2}{d}-2\right)\left(\frac{2}{d}-1\right)\left(\frac{2}{d}(\alpha-1)+1\right)} z^{-m-\frac{2}{d}} \Gamma\left(m+\frac{2}{d}\right)
\end{aligned}
$$

The products in (58) simplify as

$$
\begin{aligned}
& \prod_{j=1}^{\frac{d}{2}} \frac{\left(\frac{2}{d} j+m\right)_{3}}{2}=2^{d} d^{-\frac{3}{2} d} \frac{\Gamma\left(\frac{d}{2}(m+3)+1\right)}{\Gamma\left(\frac{d}{2} m+1\right)} \\
& \sum_{j_{l}=0}^{2} \frac{(-2)_{j_{l}}}{2 j_{l} !\left(\frac{2}{d}(l-1)+j_{l}\right)}=\frac{d^{3}}{4(l-1)(l-1+d)\left((l-1)+\frac{d}{2}\right)} \\
& \prod_{l=2}^{\frac{d}{2}-1} \frac{d^{3}}{4(l-1)(l-1+d)\left((l-1)+\frac{d}{2}\right)}=\frac{2^{2-d}(d-2)(d-1) d^{-4+\frac{3}{2} d}}{\Gamma\left(\frac{3}{2} d-1\right)}
\end{aligned}
$$

so that (58) simplifies to

$$
\frac{d \Gamma\left(\frac{d}{2}(m+3)\right)}{(d-2)(d-1)} \frac{\Gamma\left(\frac{2}{d}+m\right)(m+1)(m+2)}{\Gamma\left(\frac{d}{2} m+1\right) \Gamma\left(\frac{3}{2} d-1\right)} \frac{\frac{d}{2}(m+1)+\alpha}{\frac{d}{2}+\alpha-1} z^{-\frac{2}{d}-m} .
$$


Inserting this into Eqn. (53) we can perform the summation over $\alpha$ :

$$
\sum_{\alpha=1}^{\frac{d}{2}-1} \frac{\frac{d}{2}(m+1)+\alpha}{\frac{d}{2}+\alpha-1} \prod_{\substack{j=1 \\ j \neq \alpha}}^{\frac{d}{2}-1} \frac{j+\frac{d}{2}(m+1)}{j-\alpha}
$$

The product in the above expression gives

$$
\left(\frac{d}{2}(m+1)+\alpha\right) \prod_{\substack{j=1 \\ j \neq \alpha}}^{\frac{d}{2}-1} \frac{j+\frac{d}{2}(m+1)}{j-\alpha}=\frac{(-1)^{\alpha-1} \Gamma\left(\frac{d}{2}(m+2)\right)}{\Gamma(\alpha) \Gamma\left(\frac{d}{2}-\alpha\right) \Gamma\left(\frac{d}{2}(m+1)+1\right)}
$$

so that the sum reduces to

$$
\sum_{\alpha=1}^{\frac{d}{2}-1} \frac{(-1)^{\alpha-1} \Gamma\left(\frac{d}{2}(m+2)\right)}{\Gamma(\alpha) \Gamma\left(\frac{d}{2}-\alpha\right) \Gamma\left(\frac{d}{2}(m+1)+1\right)} \frac{1}{\alpha-1+\frac{d}{2}}=\frac{\Gamma\left(\frac{d}{2}\right)(d-1) \Gamma\left(\frac{d}{2}(m+2)\right)}{\Gamma(d) \Gamma\left(\frac{d}{2}(m+1)+1\right)} .
$$

Combining this with Eqn. (60) gives

$$
z^{-m-\frac{2}{d}} \frac{d \Gamma\left(\frac{d}{2}\right)}{(d-2) \Gamma(d) \Gamma\left(\frac{3}{2} d-1\right)} \Gamma\left(\frac{2}{d}+m\right)(m+1)(m+2)\left(\frac{d}{2}(m+1)+1\right)_{d-1}\left(\frac{d}{2} m+1\right)_{d-1} .
$$

Thus, we find

$$
\left\langle N_{m}^{d}\right\rangle(N)=\frac{N^{2-\frac{2}{d}}}{m !} \frac{\Gamma(d+1) \Gamma\left(\frac{d}{2}\right)}{(d-2) \Gamma\left(\frac{3}{2} d-1\right)} \Gamma\left(\frac{2}{d}+m\right)+ \begin{cases}\mathcal{O}(N \log N) & \text { for } d=4 \\ \mathcal{O}\left(N^{2-\frac{4}{d}}\right) & \text { for } d>4\end{cases}
$$

Realising that

$$
\frac{d \Gamma\left(\frac{d}{2}\right)}{\Gamma\left(\frac{3}{2} d-1\right)}=\frac{2}{\left(\frac{d}{2}+1\right)_{d-2}}
$$

we note that this agrees with the expression for odd dimensions, and we can thus write

$$
\left\langle N_{m}^{d}\right\rangle(N)=\frac{N^{2-\frac{2}{d}}}{m !} \Gamma\left(\frac{2}{d}+m\right) \frac{\Gamma(d)}{\left(\frac{d}{2}-1\right)\left(\frac{d}{2}+1\right)_{d-2}}+ \begin{cases}\mathcal{O}(N) & \text { for } d=3 \\ \mathcal{O}(N \log N) & \text { for } d=4 \\ \mathcal{O}\left(N^{2-\frac{4}{d}}\right) & \text { for } d>4\end{cases}
$$

for all $d>2$, which gives Eqn. (21).

\section{C.3 The case $d=2$}

$d=2$ is a special case for which ${ }_{2} F_{2}$ can be expanded for $z \rightarrow \infty$ using Mathematica,

$$
{ }_{2} F_{2}\left(\begin{array}{l}
m+1, m+1 \\
m+3, m+3
\end{array} \mid-z\right)=z^{-1-m} \Gamma(3+m)(m+1)(m+2) \log z+\mathcal{O}\left(z^{-1-m}\right) .
$$


Inserting this in 20 leads to

$$
\left\langle N_{m}^{2}\right\rangle(N)=N \log N+\mathcal{O}(N)
$$

which gives

$$
\lim _{\rho \rightarrow \infty} \frac{\left\langle N_{m}^{2}\right\rangle(\rho, V)}{\left\langle N_{0}^{2}\right\rangle(\rho, V)}=1
$$

and hence to leading order agrees with the expression for $d>2$, so that we have finally recovered Eqn. (21) for all $d \geq 2$

\section{References}

[1] L. Bombelli, J. Lee, D. Meyer, and R.D. Sorkin, "Space-Time as a Causal Set", Phys. Rev. Lett. 59:521-524 (1987).

[2] Rafael D. Sorkin. Causal sets: Discrete gravity. In A. Gomberoff, and D. Marolf, editors, Lectures on quantum gravity. Proceedings, School of Quantum Gravity, Valdivia, Chile, January 4-14, 2002, pages 305-327. Springer, January 2003.

[3] Fay Dowker. Causal sets and the deep structure of spacetime. In Abhay Ashtekar, editor, 100 Years of Relativity Space-time Structure: Einstein and Beyond. World Scientific, 2005.

[4] Sumati Surya. Directions in Causal Set Quantum Gravity. arXiv/1103.6272, 2011.

[5] Luca Bombelli, Joe Henson, and Rafael D. Sorkin. Discreteness without symmetry breaking: A Theorem. Mod.Phys.Lett., A24:2579-2587, 2009.

[6] Dionigi M.T. Benincasa, and Fay Dowker. The Scalar Curvature of a Causal Set. Phys.Rev.Lett., 104:181301, 2010.

[7] Fay Dowker, and Lisa Glaser. Causal set d'alembertians for various dimensions. Classical and Quantum Gravity, 30(19):195016, 2013.

[8] D.A. Meyer, The Dimension of Causal Sets, PhD thesis (M.I.T., 1988), G. Brightwell, and R. Gregory, "The Structure of random discrete space-time," Phys. Rev. Lett. 66:260-263 (1991), S. Major, D.P. Rideout, and S. Surya, "On Recovering continuum topology from a causal set," J.Math.Phys. 48:032501, (2007), D. Rideout, and P. Wallden, "Spacelike distance from discrete causal order," Class. Quant. Grav. 26, 155013 (2009), S. Johnston, "Particle propagators on discrete spacetime," Class. Quant. Grav. 25, 202001 (2008) , S. Johnston, "Feynman Propagator for a Free Scalar Field on a Causal Set," Phys. Rev. Lett. 103, 180401 (2009).

[9] Sumati Surya. Evidence for a Phase Transition in 2D Causal Set Quantum Gravity. Class.Quant.Grav., 29:132001, 2012.

[10] Maqbool Ahmed, and David Rideout. Indications of de Sitter Spacetime from Classical Sequential Growth Dynamics of Causal Sets. Phys.Rev., D81:083528, 2010. 
[11] E. H. Kronheimer, and R. Penrose, "On the Structure of causal spaces," Proc. Cambridge Phil. Soc. 63, 481 (1967), E.C. Zeeman, Causality Implies the Lorentz Group, J. Math. Phys. 5: 490-493, R. P. Geroch, E. H. Kronheimer, and R. Penrose, "Ideal Points In Space-Time," Proc. Roy. Soc. Lond. A 327, 545 (1972).

[12] S. W. Hawking, A. R. King, and P. J. Mccarthy, "A New Topology For Curved Space-Time Which Incorporates The Causal, Differential, And Conformal Structures," J. Math. Phys. 17, 174 (1976), D. B. Malament, J. Math. Phys. 18:1399-1404 (1977).

[13] Wolfram alpha functions. http://functions.wolfram.com/07.31.03.0010.01, August 2013.

[14] D. Kleitman, and B. L. Rothschild, Trans. Am. Math. Soc. 205, 205 (1975).

[15] Deepak Dhar. Entropy and phase transitions in partially ordered sets. J.Math.Phys., 19(8), August 1978.

[16] Deepak Dhar. Asymptotic enumeration of partially ordered sets. Pacific Journal of Mathematics, 90(2), 1980.

[17] B. Bollobas, and G. Brightwell, "The structure of random graph orders", SIAM J. Discrete Math. 10 (1997),no. 2, 318.

[18] Gabrielle Allen, Tom Goodale, Frank Löffler, David Rideout, Erik Schnetter, and Eric L. Seidel. Component specification in the cactus framework: The cactus configuration language. CoRR, abs/1009.1341, 2010.

[19] T. Goodale, G. Allen, G. Lanfermann, J. Mass, T. Radke, E. Seidel, and O. Shalf. The cactus framework and toolkit: Design and applications. Vector and Parallel Processing VECPAR'2002, 5th International Conference, Lecture Notes in Computer Science, 2003.

[20] D.P. Rideout, and R.D. Sorkin. A Classical sequential growth dynamics for causal sets. Phys.Rev., D61:024002, 2000.

[21] L. Glaser. Work in progress.

[22] Surbhi Khetrapal, and Sumati Surya. Boundary Term Contribution to the Volume of a Small Causal Diamond. Class.Quant.Grav., 30:065005, 2013.

[23] Mriganko Roy, Debdeep Sinha, and Sumati Surya. The Discrete Geometry of a Small Causal Diamond. Phys.Rev., D87:044046, 2013.

[24] A. Weil, "Sur les theoremes de de Rham", Commentarii Mathematici Helvetici 26, 119-145 (1952), Georges De Rham, "Complexes à automorphismes et homéomorphie différentiable", Annales de l'institut Fourier, 2 51-67 (1950).

[25] S. Major, D.P. Rideout, and S. Surya, "On Recovering continuum topology from a causal set," J.Math.Phys. 48:032501, (2007).

[26] Wolfram alpha functions. http://functions.wolfram.com/07.31.20.0011.01, March 2013. 